\title{
Gel Formation in Reversibly Cross-Linking Polymers
}

\author{
Maud Formanek, Lorenzo Rovigatti, Emanuela Zaccarelli, Francesco Sciortino, and Angel J. Moreno* \\ Cite This: Macromolecules 2021, 54, 6613-6627 \\ Read Online
}

ABSTRACT: By means of Langevin dynamics simulations, we investigate the gel formation of randomly functionalized polymers in solution, with the ability to form both intra- and intermolecular reversible bonds. Under highly dilute conditions, these polymers form soft nano-objects (so-called single-chain nanoparticles, SCNPs), resulting from the purely intramolecular cross-linking of the reactive functional groups. Here, we show that the competition between intra- and intermolecular bonds at finite concentration is governed by a delicate balance of various entropic contributions and leads to a density-dependent effective valence. System-
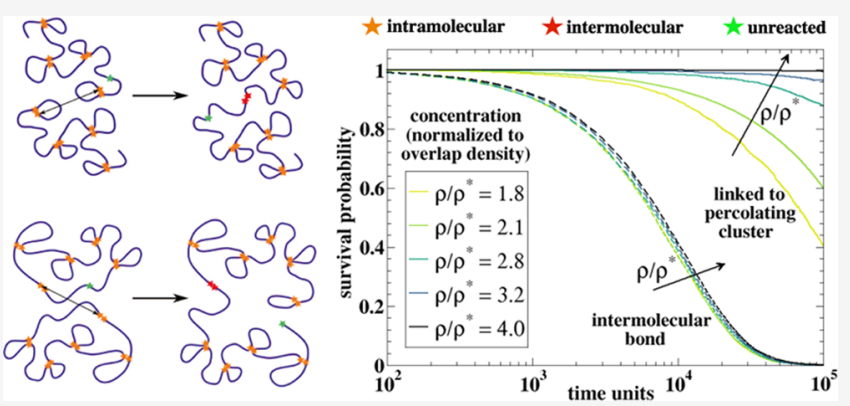
spanning networks are formed at relatively low monomer densities and their stability is mediated by just a small number of intermolecular connections per chain. The formation of intermolecular bonds furthermore can induce a nonmonotonic dependence of the polymer size on the density for long bond lifetimes. Concomitantly, the polymers in the percolating cluster adopt an intramolecular structure characteristic for self-avoiding chains, which constitutes a strong contrast to the fractal globular behavior of intramolecularly cross-linked SCNPs in crowded solutions with purely topological interactions (no intermolecular bonds). Finally, we study the dynamics of the system, which displays signatures expected for reversible gel-forming systems. An interesting behavior emerges in the reorganization dynamics of the percolating cluster: the relaxation is mostly mediated by the diffusion over long distances, through breaking and formation of bonds, of chains that do not leave the percolating cluster. Regarding the few chains that are transiently free, the time they spend until they reattach to the cluster is solely governed by the bond strength.

\section{INTRODUCTION}

Single-chain nanoparticles (SCNPs) are soft nano-objects synthesized from a linear polymer precursor, which is functionalized with reactive groups capable of forming intramolecular bonds. They are typically a few nanometers $(\leq 20 \mathrm{~nm})$ in size and possess a large surface-to-volume ratio. The necessary technological ingredients for their synthesis include controlled polymerization, monomer functionalization, and cross-linking protocols that ensure their purely intramolecular collapse. Design and function of SCNPs is a rapidly growing area of research due to their promising applications as catalysts, drug-delivery vehicles, biosensors, or rheologymodifying agents. ${ }^{1-14}$ Since its beginning at the start of the 21st century, the synthesis of SCNPs has been dominated by polymer chemistries involving irreversibly cross-linking monomers. In the past years, however, the possibility of exploiting reversible interactions to produce stimuli-responsive SCNPs has gained increased interest. ${ }^{1,2,4,5,15-18}$ Separate classes of reversible interactions with distinct advantages have emerged in the field of single-chain technology: noncovalent and the socalled dynamic covalent bonds. Noncovalent bonds are characterized by their relatively low energy (typically a few $k_{\mathrm{B}} T$ ), which is modulated smoothly by external variables such as temperature, $\mathrm{pH}$, and solvent. Prominent examples of noncovalent interactions used in SCNPs are hydrogen bonds, ${ }^{19,20}$ helical $^{21,22}$ or $\pi-\pi$ stacking, ${ }^{23}$ host-guest interactions, ${ }^{24,25}$ ionic attraction, ${ }^{26}$ and metal complex formation. ${ }^{27,28}$ In contrast to noncovalent bonds, dynamic covalent bonds are very robust and their formation, breaking, or exchange can be induced rapidly by very specific external stimuli. These can be $\mathrm{pH}$, photons, redox potentials, or a catalyst. The classical example of a dynamic covalent bond is the disulfide bridge, which plays a prominent role in the stabilization of the native folded state of proteins. It served as inspiration for including not only disulfide bonds ${ }^{29}$ but also hydrazone, ${ }^{30}$ enamine, ${ }^{31}$ coumarine, ${ }^{32}$ and anthrazene ${ }^{33}$ bonds in the SCNP chemistry toolbox.

The advantage of dynamic covalent bonds is that the need for an external stimulus to catalyze their formation and breakage opens up the possibility of kinetically trapping the system. Furthermore, reversibility means that synthesis is never "complete" and individual SCNPs of this kind can form intermolecular bonds in addition to their intramolecular bonds

Received: December 1, 2020

Revised: June 20, 2021

Published: July 7, 2021 
if their concentration is increased above the limit of high dilution. Such intermolecular bonds could potentially lead not only to aggregation or phase separation but also to the formation of a physical gel. The interplay between intra- and intermolecular bond formation has been exploited recently by Fulton et al. in thermoresponsive polymers to produce a system that reversibly crosses between an SCNP solution and a hydrogel. ${ }^{34}$ The thermoresponsive nature of the oligoethyleneglycol methyl ether branches causes the polymers to aggregate upon an increase in temperature, while a mildly acidic $\mathrm{pH}$ allows the acylhydrazone bonds to undergo component exchange processes. The combination of these two orthogonal triggers leads to the reversible reorganization of intramolecularly folded SCNPs into a robustly cross-linked hydrogel. The response of this material to multiple external stimuli could be exploited in situations where the behavior of the material should depend on the specific makeup of the environment, for example, the specific release of drugs in target tissues.

While the advances in synthesis of such reversible gels made from dynamic covalent SCNPs are promising, investigations of the structure of such materials has been lacking until now. Moreover, the theoretical description of physical gels, in general (as opposed to permanently cross-linked chemical gels), has come into focus in the soft matter community in relatively recent years. This is due in part to the difficulty of precisely defining the meaning of "gel" (systems exhibiting both dynamical arrest and network formation are generally considered as gels). A common working definition of a gel is a low-density disordered state with solid-like properties such as a yield stress. It combines properties of a liquid (through its disordered structure) and a solid (it does not flow). What distinguishes them from glasses is not only their typical low volume fraction but also their retention of quasi-ergodicity on all but the largest length scales dictated by the infinite percolating network. ${ }^{35-39}$ A second obstacle for the establishment of a unifying theoretical framework of gel formation is the lack of an ideal model system that incorporates the minimal, necessary ingredients to reproduce the universal features of a gelling system.

In Hill's formalism of liquid condensation in terms of physical clusters, phase separation induced by strong attractive interactions can be avoided by either complementing the attraction by a long-range repulsion ${ }^{40-42}$ or by modifying the attraction by limiting the valence of the interacting molecules. ${ }^{43}$ The former can be induced by excessive surface charges on colloids, ${ }^{44}$ while the latter can be achieved, for example, by decorating colloidal particles with a small number of well-defined attractive patches ${ }^{45,46}$ or the engineering of specific DNA sequences designed to form star-shaped architectures with sticky ends. ${ }^{47,48}$ An advantage of such limited-valence particles lies in the possibility of theoretically calculating their free energy within the formalism of Wertheim theory, ${ }^{49,50}$ which allows one to determine the phase diagram of the system. ${ }^{51}$ Furthermore, the increased experimental control over such patchy particles achieved in the past decade has paved the way for their use as highly tunable building blocks for the design of self-assembled materials. ${ }^{52,53}$

SCNPs with reversible bonds may display characteristics of both microphase separating colloids and patchy particles due to the competition between intra- and intermolecular bonds. At very high dilution, intermolecular bonds should be disfavored by the long intermolecular distances. Upon increasing the volume fraction, some of the intramolecular bonds will be exchanged for connections with other chains for entropic reasons, possibly forming a system-spanning network for the right combination of system parameters. We expect phase separation of the system to be confined to very small densities through the combination of excluded volume interactions and the inherently limited "valence" of the polymers that originates from the locally small number of (monovalent) monomers capable of forming bonds. With these ideas in mind, in this article, we present Langevin dynamics simulations of solutions of a bead-spring model for SCNPs with reversible bonds, exploring concentrations from high dilution to far beyond the overlap density. We characterize the structural and dynamic changes produced by the competition between intra- and intermolecular bonding as the concentration increases. We find that the number of intramolecular bonds is not constant but decreases with the concentration, which complicates the possibility of considering the SCNPs as objects with a characteristic valence, and thus of straightforwardly applying Wertheim's formalism. A systemspanning cluster is formed above the overlap concentration. The scaling of the cluster size distribution is consistent with nonmean field critical percolation. The connectivity of the percolating cluster is mediated by a few intermolecular connections per chain. In contrast with the fractal ("crumpled") globular conformations adopted by purely intramolecularly cross-linked SCNPs (no intermolecular bonds) under purely steric crowding and topological constraints, ${ }^{54}$ intermolecular bonding in the dense solutions just leads to weak perturbations with respect to the limit of high dilution. The analysis of the dynamics reveals that, in analogy to the case of colloids with limited valence, the system forms a reversible gel with no apparent signatures of phase separation in the broad range of investigated densities. Relaxation of the dynamic network is mostly mediated by diffusion, through breaking and formation of bonds, of chains that, without detaching from the percolating cluster, are able to explore distances of several times their size. The article is organized as follows. Section 2 presents the model and simulation details. Section 3 shows and discusses structure and dynamics in the scenario emerging as the concentration increases and the system changes from a dilute solution of SCNPs to a reversible gel. Conclusions are given in Section 4.

\section{SIMULATION DETAILS}

The polymers are simulated as chains of beads and springs according to the model of Kremer and Grest. ${ }^{55}$ As such, they represent uncrossable flexible chains with excluded volume interactions under implicit good solvent conditions, and the bead size $\sigma$ qualitatively corresponds to a Kuhn length ${ }^{56}(\sigma \lesssim 1$ $\mathrm{nm}$ ). The chains consist of $N=200$ bead ("monomers"), consecutively linked together by irreversible backbone bonds modeled via the FENE potential ${ }^{55}$

$$
U^{\mathrm{FENE}}(r)=-\varepsilon K_{\mathrm{F}} R_{0}{ }^{2} \ln \left[1-\left(\frac{r}{R_{0}}\right)^{2}\right]
$$

with $K_{\mathrm{F}}=15$ and $R_{0}=1.5$. Monomer excluded-volume interactions are given by a purely repulsive Lennard-Jones potential (Weeks-Chandler-Andersen potential) 


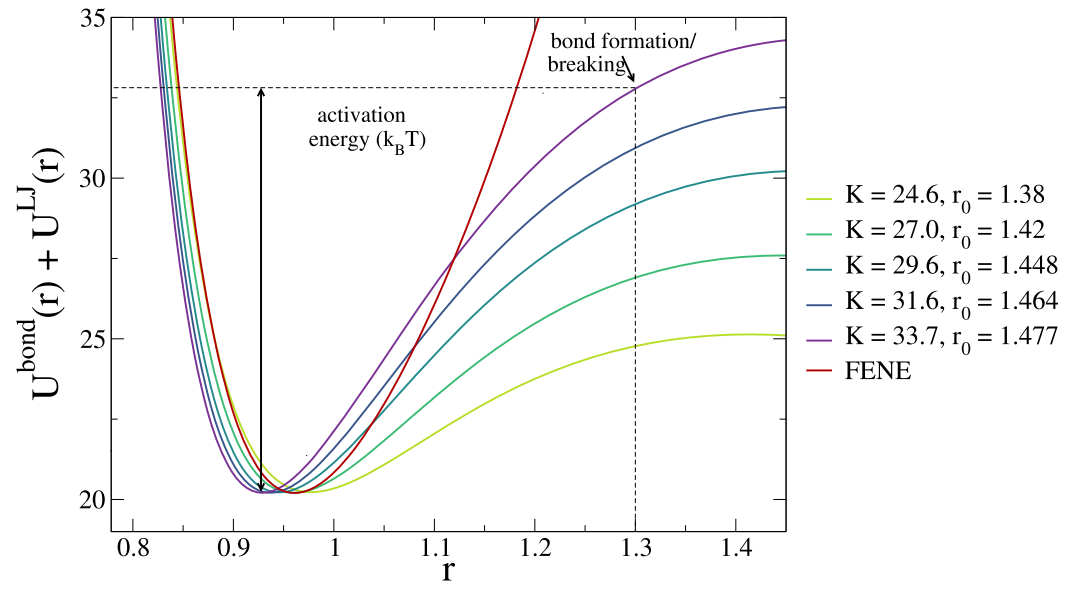

Figure 1. Sum of the bonding ( $U^{\text {bond }}=U^{\mathrm{FENE}}$ for irreversible bonds or $U^{\mathrm{rev}}$ for reversible ones) and monomer excluded-volume $\left(U^{\mathrm{LJ}}\right)$ potentials. Data are shown for different bond strengths $K$ and for the irreversible (FENE) case.

$$
U^{\mathrm{LJ}}(r)=4 \varepsilon\left[\left(\frac{\sigma}{r}\right)^{12}-\left(\frac{\sigma}{r}\right)^{6}+\frac{1}{4}\right]
$$

with a cutoff distance $r_{\mathrm{LJ}}=2^{1 / 6} \sigma$, at which both the potential and the corresponding forces are continuous. The sum of both potentials leads to a deep minimum at $r_{\min }=0.96 \sigma$ for the total interaction between two bonded beads, which limits the bond fluctuations and guarantees uncrossability. The units of energy, length, mass, and time are, respectively, $\varepsilon, \sigma, m$, and $\tau=\sqrt{\sigma^{2} m / \varepsilon}$. In the rest of the article, all numerical values will be given in reduced units $\varepsilon=\sigma=m=\tau=1$.

A fraction $f=N_{\mathrm{r}} / N$ of the monomers is randomly chosen to be of the reactive type, which can form, as in the standard experimental routes, monofunctional reversible bonds with other reactive monomers, that is, if a reversible bond connects two reactive monomers, such monomers are not allowed to form new bonds until the former breaks. A bond is formed whenever two unbonded reactive monomers approach each other in space and are separated by less than the capture radius $r_{\mathrm{c}}=1.3$. If there are several candidates within the capture radius, a random choice is made. To avoid trivial bonding, the random distribution of reactive monomers along the chain backbone is made with the constraint that any two consecutive reactive monomers are separated by at least one nonreactive monomer. This bond formation is identical to the cross-linking process in the case of irreversibly intramolecularly cross-linked SCNPs employed in previous studies. ${ }^{54,57-60}$ Once a bond is formed, the two participating monomers interact via a Morse potential

$$
U^{\mathrm{rev}}(r)=K \varepsilon\left[2 \mathrm{e}^{\left(r_{0}-r\right)}-\mathrm{e}^{2\left(r_{0}-r\right)}\right]
$$

with adjustable parameters $K$ and $r_{0}$. In the case of the irreversible cross-linking, if two reactive monomers form a bond, they interact for the rest of the simulation via the same FENE potential (eq 1) as the permanent backbone bonds.

Reversible bonds can be broken again if, at any given time step, the participating monomers are separated by a distance $r$ $>r_{\mathcal{c}}$ upon which their interaction via the Morse potential terminates. The parameter $K$ governs the bond strength through modulating the energy barrier that has to be overcome in order to break the bond. Since $r_{\mathrm{c}}>r_{\mathrm{LJ}}$ and hence $U^{\mathrm{LJ}}\left(r_{\mathrm{c}}\right)=0$, the barrier is given by the energy difference $U^{\text {rev }}\left(r_{c}\right)-U_{\text {min }}$, where $U_{\text {min }}$ is the minimum of $U^{\mathrm{LJ}}+U^{\mathrm{rev}}$. As such, bond formation is independent of $K$, while bond breakage depends on $K$. Thus, varying $K$ does not only change the average bond lifetime but also change the average probability of any reactive monomer being bonded at equilibrium. The remaining free parameter $r_{0}$ is chosen such that the minimum $U_{\min }$ of the sum of the nonbonded and bonded interactions is, with respect to the irreversible case, the same in the energy and as close as possible in the distance. Just a slight variation in the range 0.93 $<r_{\text {min }}<0.98$ is obtained, see Figure 1 . This leads to a slight difference in the effective monomer excluded volume. For the range of investigated monomer concentrations (much lower than melt densities, see below) such a slight difference is irrelevant and does not affect the qualitative physical scenario (see Supporting Information). Still, one should note that small differences in $r_{\min }$ would be important in a melt (specially if it is close to dynamic arrest), where excluded volume would compete with the bonding dynamics.

Contrary to patchy particle models, in which the monofunctionality of the bonds is encoded in the geometry of the interaction, ${ }^{48,51,61,62}$ we enforce monofunctionality by keeping a list of bonded pairs. Reactive monomers that are mutually bonded cannot form other bonds until their mutual bond is broken (i.e., until their mutual distance becomes $r>$ $\left.r_{\mathrm{c}}\right)$. We perform Langevin dynamics simulations at a fixed temperature $T=\varepsilon / k_{\mathrm{B}}=1$ (with $k_{\mathrm{B}}$ being the Boltzmann constant). We use a time step $\Delta t=0.01$ and a friction coefficient $\gamma=0.05$ (which achieves good thermalization without delaying the dynamics significantly). The equations of motion are integrated following the scheme of ref 63 . Before simulating reversibly cross-linking chains at various densities, we perform exploratory simulations of isolated single chains (mimicking the limit of infinite dilution), for various values of $f$ (fraction of reactive groups) and $K$ (bond strength). We calculate, for such isolated chains, the bond probability $p_{\mathrm{B} 0}$, which is defined as the ratio between the average number of formed bonds and the maximum number of bonds (corresponding to the fully cross-linked SCNP). We also characterize the size and shape of the isolated SCNPs by determining the gyration ellipsoid and, from it, the radius of gyration $\left(R_{\mathrm{g} 0}\right)$, asphericity $\left(a_{0}\right)$, and prolateness $\left(p_{0}\right)$ parameters. ${ }^{64^{8}}$ The asphericity $0 \leq a \leq 1$ quantifies deviations of the gyration ellipsoid from the spherical shape (corresponding to $a=0)$. The prolateness varies between the limits of perfectly prolate $(p=1)$ and oblate $(p=-1)$ ellipsoid. The 
results are summarized in Table 1 . Increasing the bonding probability through the increase in $K$ or $f$ leads to smaller, more

Table 1. Radius of Gyration $R_{\mathrm{g} 0}$, Asphericity $a_{0}$, Prolateness $p_{0}$, and Bonding Probability $p_{\mathrm{B} 0}$, as a Function of the Bond Strength $K$ and the Fraction of Reactive Groups $f$, under Highly Dilute Conditions $(\rho \rightarrow 0)^{a}$

\begin{tabular}{rlrrrrrc}
$f$ & \multicolumn{1}{c}{$K$} & $r_{0}$ & $R_{\mathrm{g} 0}$ & \multicolumn{1}{c}{$a_{0}$} & \multicolumn{1}{c}{$p_{0}$} & \multicolumn{1}{c}{$p_{\mathrm{B} 0}$} & $\rho^{*}$ \\
0.0 & linear chains & & 11.4 & 0.446 & 0.740 & 0 & 0.017 \\
0.1 & 24.6 & 1.380 & 11.3 & 0.444 & 0.739 & 0.27 & 0.017 \\
& 27.0 & 1.420 & 10.9 & 0.443 & 0.735 & 0.47 & 0.019 \\
& 29.6 & 1.448 & 10.4 & 0.435 & 0.727 & 0.70 & 0.022 \\
& 31.6 & 1.464 & 9.9 & 0.426 & 0.726 & 0.83 & 0.026 \\
& 33.7 & 1.477 & 9.3 & 0.411 & 0.720 & 0.91 & 0.031 \\
& irreversible & & 8.0 & 0.338 & 0.634 & 1 & 0.049 \\
0.2 & 24.6 & 1.380 & 11.0 & 0.445 & 0.739 & 0.48 & 0.019 \\
& 27.0 & 1.420 & 10.4 & 0.438 & 0.733 & 0.68 & 0.022 \\
& 29.6 & 1.448 & 9.8 & 0.432 & 0.728 & 0.84 & 0.027 \\
& 31.6 & 1.464 & 9.4 & 0.423 & 0.718 & 0.92 & 0.030 \\
& 33.7 & 1.477 & 8.8 & 0.406 & 0.658 & 0.96 & 0.037 \\
& irreversible & & 7.7 & 0.347 & 0.658 & 1 & 0.055 \\
0.3 & 24.6 & 1.380 & 10.7 & 0.444 & 0.727 & 0.60 & 0.020 \\
& 27.0 & 1.420 & 10.2 & 0.440 & 0.752 & 0.78 & 0.024 \\
& 29.6 & 1.448 & 9.7 & 0.440 & 0.744 & 0.90 & 0.027 \\
& 31.6 & 1.464 & 9.2 & 0.423 & 0.733 & 0.95 & 0.032 \\
& 33.7 & 1.477 & 8.5 & 0.411 & 0.719 & 0.97 & 0.041 \\
& irreversible & & 7.5 & 0.352 & 0.659 & 1 & 0.059
\end{tabular}

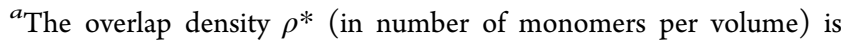
included. The values of all the former quantities for irreversibly crosslinked SCNPs and for simple unbonded linear chains are included for comparison.

spherical and less prolate SCNPs. In all cases, $a_{0}$ is far from zero and the obtained SCNPs have, on average, sparse topologies, as will be confirmed by the analysis of the form factors (see below).

Since, in the crowded solutions, we are interested in exploring the possibility of forming gels from the reversibly cross-linking chains, the bonding probability needs to be high enough for a system-spanning cluster to form and give the material the ability to propagate stress throughout the whole system. On this basis, we choose two fractions of reactive monomers $f=0.1$ and 0.3 that guarantee a high number of potential sites for forming intermolecular bonds, and values of the bond strength parameters that are high enough to stabilize the percolating network $(K=29.6$ and 33.7, corresponding to activation energies of about $9 k_{\mathrm{B}} T$ and $13 k_{\mathrm{B}} T$, see Figure 1). Moreover, the choice of different values of the bond activation energy and fraction of reactive groups allows us to test the consistency of the emerging qualitative physical scenario. The number of reactive groups in the chain of $N=200$ monomers for the former values of $f$ is even so that in the limit of fully intramolecularly cross-linked SCNPs, there are no unbonded reactive groups.

In the solutions of reversible chains, we keep the total number of polymers $N_{\mathrm{p}}=108$, and thus, the total number of monomers $N_{\mathrm{m}}=N_{\mathrm{p}} N=21,600$, fixed for all systems. We vary the side $L_{\text {box }}$ of the cubic simulation box to obtain the desired values of the monomer density $\rho=N_{\mathrm{m}} / L_{\mathrm{box}}{ }^{3}$. The behavior of these systems will be compared with previous simulations of solutions of irreversible SCNPs. ${ }^{54,65}$ For these simulations, $N_{\mathrm{p}}$ $=200$ topologically polydisperse, purely intramolecularly cross- linked SCNPs with permanent bonds were first generated. Solutions of such 200 SCNPs were prepared by placing them in the simulation box, preventing concatenations, and slowly compressing the box to different target concentrations, followed by equilibration. Since, by construction, the intramolecular bonds in the irreversible SCNPs were permanent and no intermolecular bonds were allowed, the intermolecular interactions in these solutions were just given by excluded volume and noncrossability of the chain segments (topological constraints). These interactions are at the origin of the collapse of the irreversible SCNPs to crumpled globular conformations in crowded solutions, ${ }^{54}$ instead of the milder transition from self-avoiding to Gaussian conformations adopted by simple unbonded linear chains. In what follows, data sets for these systems will be denoted as "intramolecular" to stress that all bonds are intramolecular by construction and intermolecular bonds are absent. For comparison, we also simulated solutions of simple linear chains without reactive groups and with the same $N=200$. Further details of the simulations of the intramolecularly cross-linked SCNPs and the linear chains can be found in refs 54and. ${ }^{65}$ Their gyration radii and shape parameters at $\rho \rightarrow 0$ are included in Table 1.

For the remainder of the article, the reversible systems will be denoted by their values of $K$ and $f$. The density of the system will be given in reduced units $\rho / \rho^{*}$, where $\rho^{*}$ is the overlap concentration. We define this quantity as $\left(\rho^{*}=-\right.$ $\left.2 R_{\mathrm{g} 0}\right)^{-3}$, with $R_{\mathrm{g} 0}$ being the radius of gyration of the isolated polymer $(\rho \rightarrow 0$, see Table 1$)$. We expect intermolecular crosslinks to begin forming significantly around the overlap concentration, when monomers of different chains start to enter the same space. Our definition of $\rho^{*}$ is pragmatic: it describes the concentration at which simple (nonreactive) linear chains start to shrink better than the usual definition based on a sphere of radius $R_{\mathrm{g}}$ containing the monomers of a single macromolecule. ${ }^{54}$

We explore densities in the range $0 \leq \rho / \rho^{*} \leq 4$.4. This corresponds to monomer densities up to $\rho \approx 0.14$, far below the melt density $(\rho \approx 1)$. The investigated densities are also below the entanglement density for linear chains of the same polymerization degree, $\rho_{\mathrm{e}} \gtrsim 0.42$. The latter is calculated as ${ }^{56}$ $\rho_{\mathrm{e}}=\left(N_{\mathrm{e}} / N\right)^{3 \nu_{\mathrm{F}}-1}$, where $\nu_{\mathrm{F}}=0.588$ is the Flory exponent and $N_{\mathrm{e}}$ is the entanglement length of linear chains in the melt state. For the simulated bead-spring model, a value $N_{\mathrm{e}} \gtrsim 65$ is found. ${ }^{66}$ For each combination $(K, f)$ and each density, eight independent simulation runs were carried out, each consisting of $1 \times 10^{7}$ equilibration steps and $4 \times 10^{7}$ production steps. The total CPU time used in the simulations was about 400,000 h.

\section{RESULTS AND DISCUSSION}

Competition between Intra- and Intermolecular Bonds. Ideally, one would wish to derive a thermodynamic description of the system, for example, according to Wertheim theory, ${ }^{49,50}$ using inputs from computer simulations, which would allow us to evaluate the complete $K-\rho$ phase diagram of these reversibly cross-linking polymers and to find the regions in which gel formation is possible. Wertheim thermodynamic perturbation theory (TPT) was originally developed for associating liquids but has also been successfully employed to elucidate the phase behavior of gel-forming systems of limited valence qualitatively and sometimes even quantitatively reproducing numerical results. ${ }^{51,67,68}$ 
A few fundamental assumptions of TPT have to be satisfied in order to be able to describe the system according to its predictions: (i) bonds are strictly monofunctional, (ii) two molecules cannot share more than one bond, and (iii) molecules cannot form bonds with themselves. While the assumption (ii) might be problematic at very high densities, (iii) is inherently violated in a flexible polymeric molecule with many functional groups along its backbone. However, if the number of intramolecular bonds stays approximately constant at different densities, we can neglect intramolecular bonds and view the polymers as having an "effective" valence of $M=[1-$ $\left.p_{\mathrm{B}}\right] N f$. If this is the case, the polymers might behave similar to patchy particles but with the distinction that the "patches" are not located at specific points on their surface, but randomly distributed, and fluctuate due to the inherent softness of the polymer and the rearrangement of the intramolecular bonds along the chain backbone. In this view, the intramolecular bonds solely affect the reference free energy.

We test whether the assumption of the intramolecular bonds being unaffected by interactions with other polymers holds over a certain range of densities. Figure 2 a displays the loss of
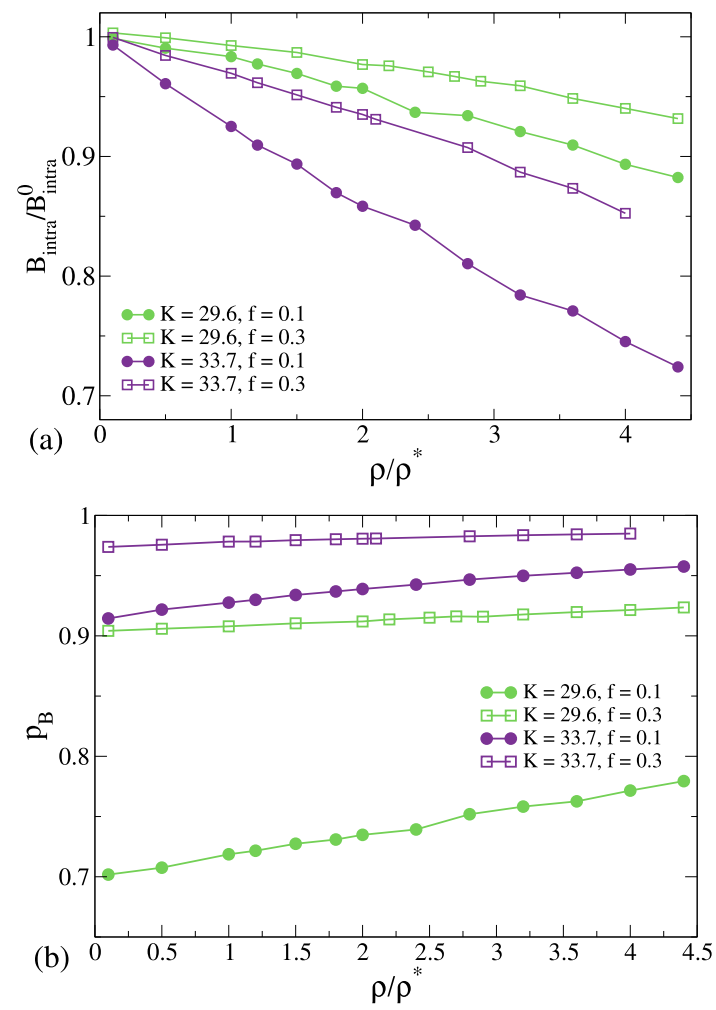

Figure 2. (a) Average number $\left(B_{\text {intra }}\right)$ of intramolecular bonds per molecule normalized by its value at infinite dilution $\left(B_{\text {intra }}^{0}\right)$, for different values of the energy constant $K$ and reactive monomer fraction $f$, as a function of the density. (b) Total (sum of intra- and intermolecular) bonding probability $p_{\mathrm{B}}$ for different values of $(K, f)$, as a function of the density.

intramolecular bonds per chain as a function of density. For all $(K, f)$ parameter combinations, increasing the density of the system leads to a competition between intra- and intermolecular bonds, instead of a simple addition of intermolecular bonds to the SCNP. The effect is the strongest for $K=33.7$ and $f=0.1$, which shows a loss of about $30 \%$ of the intramolecular bonds by going from infinite dilution to high concentration $\rho / \rho^{*} \sim 4$. Figure $2 \mathrm{~b}$ shows the total (intra- and intermolecular) bonding probability, $p_{\mathrm{B}}$, as a function of the density. The parameter combination with the lowest bonding probability at high dilution, $K=29.6$ and $f=0.1$, exhibits the strongest increase in $p_{\mathrm{B}}$. Here, the loss in entropy stemming from crowding of the surrounding molecules can be largely compensated by the enthalpic gain of forming new additional intermolecular bonds. On the other hand, for $K=33.7$ and $f=$ 0.3 , the bonding probability is already saturated at low density and stays almost constant upon crowding the system.

Since systems with $K=33.7$ form bonds more easily than those with $K=29.6$, it is not surprising that at the same reduced density $\rho / \rho^{*}$, they also form more intermolecular bonds and hence show a higher loss of intramolecular bonds. A less intuitive finding is that at fixed $K$ and $\rho / \rho^{*}$, increasing the fraction of reactive groups $f$ leads to a lower loss of intramolecular bonds. Although having more available reactive groups facilitates, in absolute terms, the formation of more intermolecular bonds (as shown later in Figure 5), in relative terms, the loss of intramolecular bonds is lower. This occurs because by increasing $f$, reactive groups become closer along the backbone, which leads to frequent exchange events that do not alter the number of intramolecular bonds. Finally, since Figure $2 \mathrm{a}$ demonstrates that the number of intramolecular bonds is not constant but strongly decays as the density grows, we conclude that a straightforward application of Wertheim's theory is not possible in the present case.

The competition between intra- and intermolecular bonds when the system is highly bonded has to be understood in terms of a delicate interplay between various entropic and energetic contributions to the free energy of the system. One might expect that the formation of intramolecular bonds is favorable over the formation of connections with other molecules, as the latter reduces the translational entropy of both molecules without a compensating energetic gain (the bonds are energetically equivalent). On the other hand, depending on the contour length separating the monomers whose intramolecular bond is exchanged for an intermolecular bond, it might be that the breakage of the former could potentially increase the conformational entropy of the molecule that loses the intramolecular link. Figure 3 presents an example of two possible bond recombination events. In scenario (a), two intramolecular bonds between monomers separated by short contour distances are exchanged for an intermolecular bond (and a different intramolecular bond to keep the number of free reactive groups constant). The conformational entropy is thus only mildly affected by the breakage of the intramolecular bonds, while the translational entropy is decreased significantly, as the two molecules now have to diffuse together. In scenario (b), each separate molecule contains a long-range loop formed by the connection of its two backbone ends. The opening of this loop via the exchange for an intramolecular bond increases the conformational entropy of both molecules, as one of their ends (the one not participating in the newly formed intermolecular bond) becomes floppier and acquires increased freedom to explore different conformations. Apart from the entropic contributions of increasing or decreasing conformational and translational degrees of freedom, one has to consider the purely combinatorial increase in entropy due to the possibility of forming intermolecular bonds. ${ }^{69,70}$ This delicate interplay between the various entropic contributions should be at the origin of the loss of intramolecular bonds in favor of 

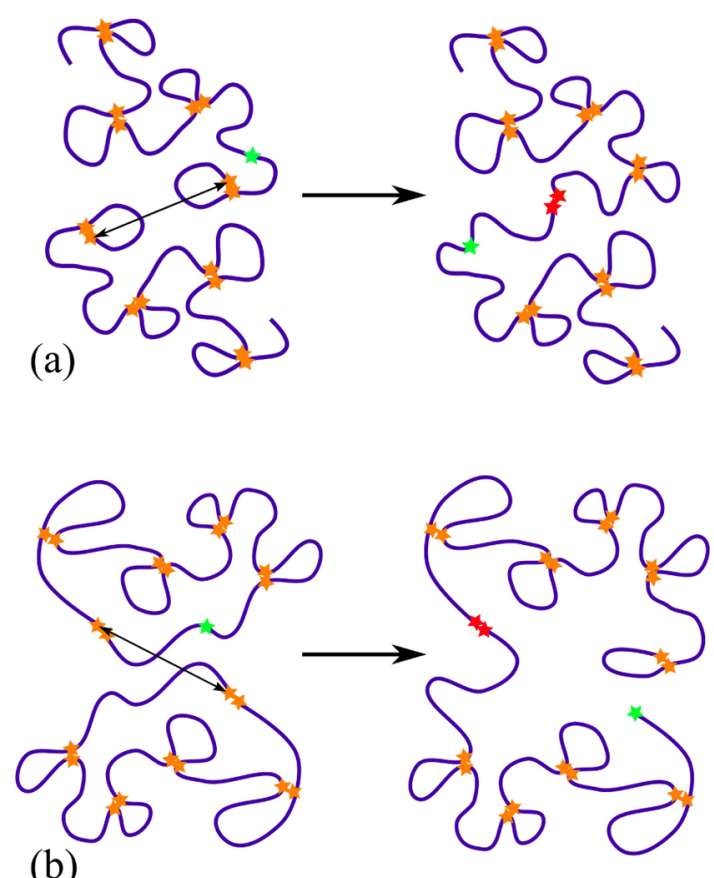

Figure 3. Schematic examples of two possible recombinations between intramolecular (orange stars) and intermolecular (red stars) bonds. Both the number of free reactive monomers (green stars) and the total number of bonds remain unchanged in both events. (a) Two short intramolecular loops are exchanged for an intermolecular bond; (b) two long intramolecular loops break through the formation of an intermolecular bond. The total number of bonds remains unchanged in both (a) and (b) through the additional formation of a small intramolecular loop.

energetically equivalent intermolecular bonds when the concentration increases and renders a treatment within Wertheim theory impossible without significant modifications of the theory.

Intermolecular Bonding and Percolation. A necessary, albeit not sufficient, prerequisite for gelation is the emergence of a network of bonds spanning the whole system in all three directions. In chemical gels, where intermolecular bonds are irreversible, the onset of this percolation coincides with the system acquiring a finite shear modulus and an infinite zeroshear viscosity (the gel stops flowing). In physical gels, where bonds are transient, clusters can break and reform over time, which strongly affects the viscoelastic properties of the system. Thus, the transient appearance, or even the persistence in time, of a system-spanning cluster in physical gels does not guarantee the propagation of external stresses throughout the whole system for all time scales or the occurrence of dynamical arrest, which takes place at concentrations/temperatures beyond the percolation point.

We explore the formation of a spanning cluster in our system. Figure 4 displays the distribution of cluster sizes for the whole range of densities and all the combinations of the parameters $K$ and $f$. Here, two chains belong to the same cluster if they are mutually connected by at least one intermolecular bond. The maximum cluster size $N_{\text {cluster }}^{\max }$ is 108 , that is, the total number of polymers in the simulation box, which has been kept fixed for all the concentrations. Irreversible gelation processes are well-described by the FloryStockmayer (FS) mean-field theory of percolation if two conditions are met: the bonds are independent from each

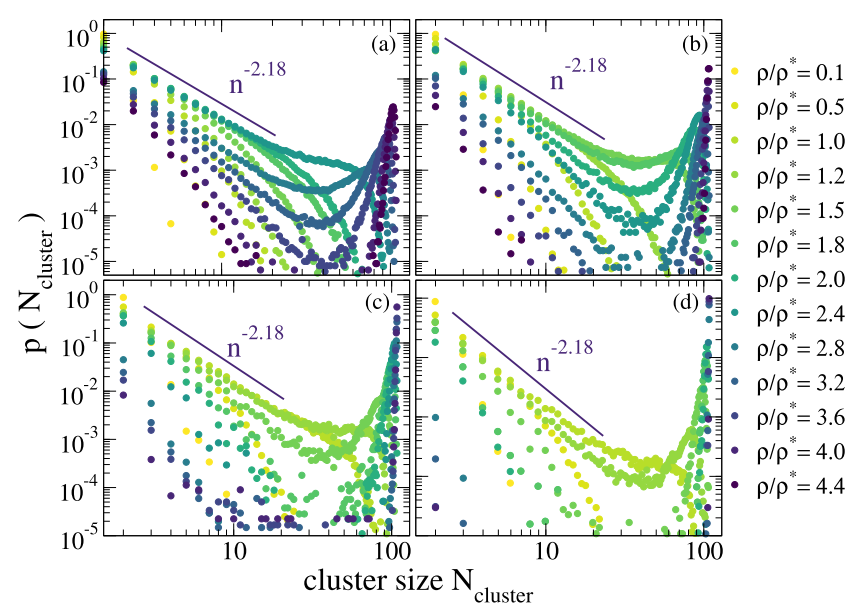

Figure 4. Cluster size distribution for $K=29.6(\mathrm{a}, \mathrm{b})$ and $K=33.7$ (c,d) with $f=0.1(\mathrm{a}, \mathrm{c})$ and $f=0.3(\mathrm{~b}, \mathrm{~d})$ for various densities (see legend). Solid lines are power laws $p\left(N_{\text {cluster }}\right) \sim N_{\text {cluster }}{ }^{-2.18}$.

other and loops are not present in the system..$^{71-73}$ Under these assumptions, if the functionality of the monomers is known, the percolation threshold can be calculated in terms of a critical bond probability $p_{\mathrm{B}}^{\mathrm{c}}$, which depends on the temperature or attraction strength and on the volume fraction. $^{71,74}$ Close to this critical point, the cluster size distribution follows a power law $p\left(N_{\text {cluster }}\right) \sim N_{\text {cluster }}{ }^{-\tau}$ where for real (nonmean-field systems), $\tau \approx 2.18$. As can be seen in Figure 4, each combination of $K$ and $f$ has a concentration range for which the initial part of the cluster size distribution is well-described by a power law with the expected exponent $\tau \approx$ 2.18 .

Since the "effective valence" of these reversibly cross-linking polymers is dependent on the density, reactive monomer fraction, and bond strength, we are interested to know in how many intermolecular bonds a chain forms on average as the system starts to percolate. One has to keep in mind, however, that multiple bonds are possible between two specific polymers, and every additional bond shared between two given chains does not add to the overall connectivity of the network. In Figure 5a, we thus display both the average number of intermolecular bonds per chain as well as the average connectivity $C$ (i.e., to how many different chains is a given chain connected through interchain bonds, irrespective of the number of bonds mediating the connection between each pair of chains). The values at which a pronounced peak at large cluster sizes first appears in $P\left(N_{\text {cluster }}\right)$ (see Figure 4$)$ are marked by ellipses in Figure 5a. These values provide an estimation of the percolation line and, as can be seen, this occurs at densities above and even far above the overlap concentration. The general trend is that the percolation transition approaches the overlap concentration from above by increasing the fraction of reactive groups and the bond strength.

Figure $5 \mathrm{a}$ shows that the average connectivity is $C \approx 2$ around the percolation transition. We calculate the ratio $B_{\text {inter }} /$ $C$, that is, how many intermolecular bonds are formed on average between two mutually connected chains (Figure 5b). Interestingly, increasing the concentration does not lead to a higher number of bonds per pair of connected chains. The number of interchain connections increases but the average number of bonds mediating a connection, $B_{\text {inter }} / C$, stays approximately constant across the whole range of densities for 

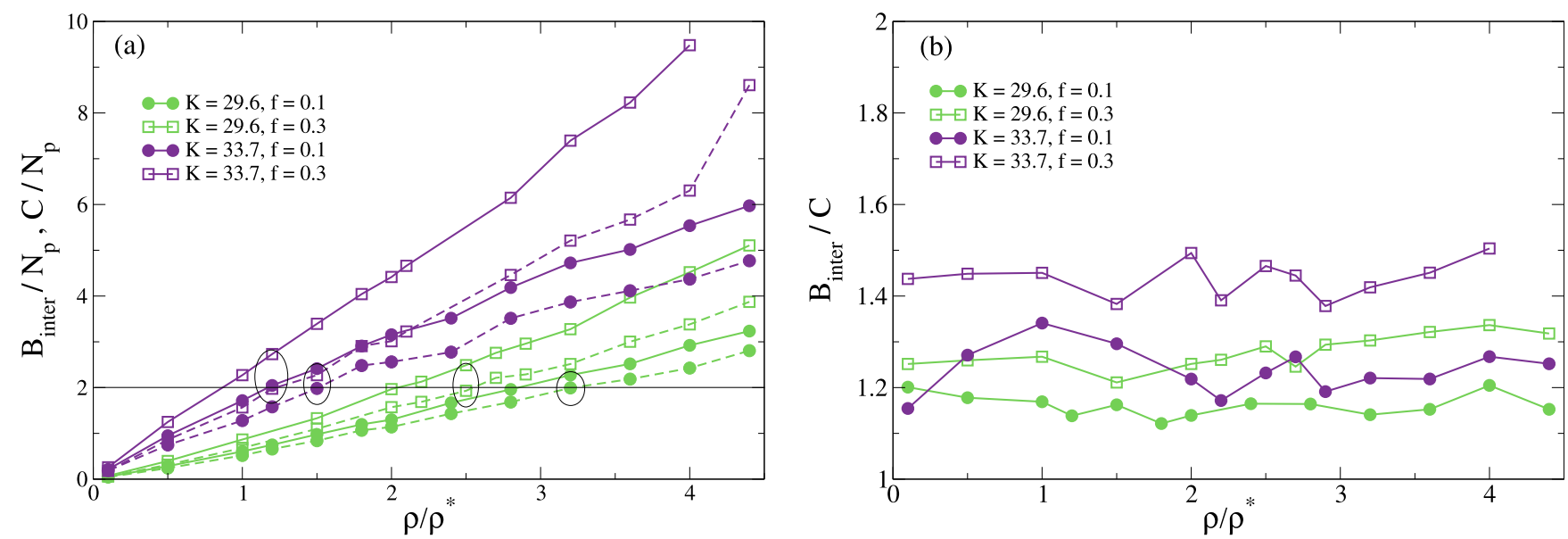

Figure 5. (a) Average number of intermolecular bonds ( $B_{\text {inter }}$, solid lines) and connectivity ( $C$, dashed lines) per molecule for different values of the energy constant $K$ and reactive monomer fraction $f$, as a function of the density. The values at which a pronounced peak at large cluster sizes first appears in $P\left(N_{\text {cluster }}\right)$ (see Figure 4$)$ are marked by ellipses. (b) Average number of intermolecular bonds $B_{\text {inter }}$ per connection with a different molecule, for different values of the energy constant $K$ and reactive monomer fraction $f$, as a function of the density.
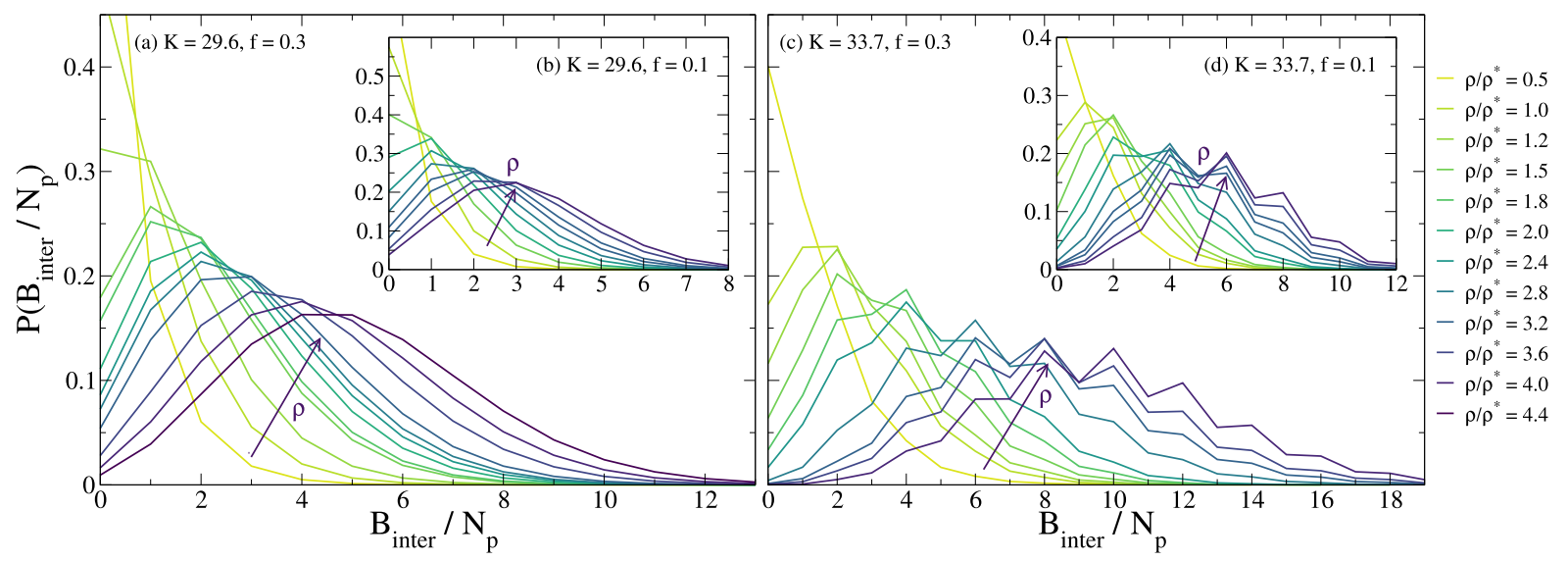

Figure 6. Distribution of intermolecular bonds per chain for $K=29.6(\mathrm{a}, \mathrm{b})$ and $K=33.7$ (c,d) for various densities. The main panels depict the case $f=0.3(\mathrm{a}, \mathrm{c})$, while the insets represent the case $f=0.1(\mathrm{~b}, \mathrm{~d})$. The arrows indicate the direction of increasing density (also see legend).

all the $(K, f)$ parameter combinations. Higher fractions of reactive monomers $f$ lead to higher values of $B_{\text {inter }} / C$, as expected. In all cases, an average of about $1.2-1.4$ intermolecular bonds mediating an interchain connection is sufficient to mantain the spanning network.

To gain a deeper understanding of the formation of intermolecular bonds, we next investigate the distribution of intermolecular bonds at specific densities. Figure 6 displays the distributions for $K=29.6$ and $K=33.7$, the main panels presenting the case $f=0.3$ and the insets presenting the case $f$ $=0.1$. The distributions reveal a difference between the energy landscapes of the systems for both bond strengths, whereas for $K=29.6$, the distributions are smooth with a clear maximum, the data for $K=33.7$ exhibit a characteristic zig-zag pattern, with even values of $B_{\text {inter }}$ being more favorable than their closest odd values. This feature can be understood as follows. Since, as mentioned in Section 2, the total number of reactive groups in the chain is even, forming an odd number of intermolecular bonds meaning that at least one reactive monomer of the chain remains unbonded. On the other hand, the number of unreacted monomers is very low for $K=$ 33.7 since indeed $p_{\mathrm{B}}$ is very close to the full-bonding limit $p_{\mathrm{B}}=$ 1 (Figure 2). In this limit of saturation, the penalty in energy and combinatorial entropy is high enough to disfavor odd numbers of intermolecular bonds per chain over even ones.

Structural Properties. In the previous section, we have shown that the intramolecular bonds are not unaffected by the presence of other molecules, but rather that intra- and intermolecular bonds compete with each other, the outcome of which depends on a delicate interplay of various entropic contributions. We expect this exchange of intra- for intermolecular bonds to be accompanied by structural changes in the polymers. The partial unfolding induced by the opening of intramolecular loops might to some degree counteract the collapse found above the overlap concentration in the case of the irreversibly intramolecularly cross-linked SCNPs. A first measure of structural changes upon increasing the density is the size of the polymers, given by the radius of gyration depicted in Figure 7a. We find that the competition between steric repulsion and partial unfolding leads to qualitatively different density dependences of the molecular size. For $K=$ 29.6, shrinking due to macromolecular crowding dominates, whereas for the strong association energy $K=33.7$, the polymers swell slightly with respect to their conformations at high dilution. At high densities, however, a re-entrance of $R_{g}$ for $K=33.7$ can be observed. For comparison, we include the corresponding data for linear chains without reactive groups 

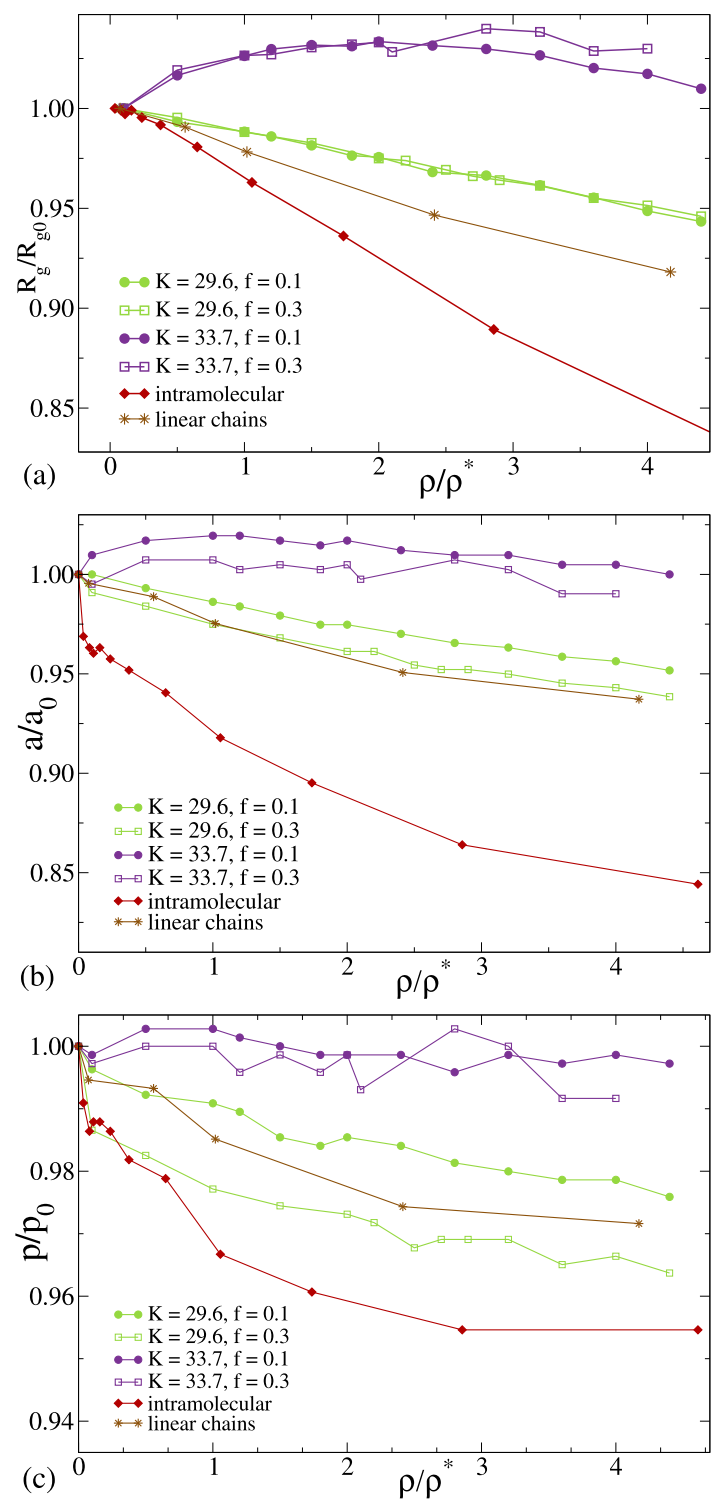

Figure 7. Normalized radius of gyration (a), asphericity (b), and prolateness (c) for different values of the energy constant $K$ and reactive monomer fraction $f$, as well as for intramolecularly crosslinked SCNPs (no intermolecular bonds) and simple linear chains without reactive groups, as a function of the density. and for the intramolecularly cross-linked SCNPs (no intermolecular bonds). In both cases, shrinking is stronger than in the reversible systems. The effect is especially pronounced in the intramolecularly cross-linked SCNPs, resembling the observation of ring polymers collapsing to crumpled globular structures. ${ }^{54,65,73}$ Panels (b) and (c) of Figure 7 show the corresponding results for the asphericity and prolateness parameters, respectively. The observed trends are qualitatively similar to those of the radius of gyration.

A more detailed description of the intramolecular structure of the polymers is given by their isotropic form factors

$$
w(q)=\left\langle\frac{1}{N} \sum_{j, k} \frac{\sin \left(q r_{j k}\right)}{q r_{j k}}\right\rangle
$$

where $r_{j k}$ is the distance between monomers $j$ and $k$ and the sum only includes pairs of monomers belonging to the same molecule. Being fractal objects, the form factors of polymers typically follow a scaling law $w(q) \sim q^{-1 / \nu}$ for wave vectors corresponding to length scales bigger than the bond length $b$ but smaller than the radius of gyration, that is, $1 / R_{g} \lesssim q \lesssim 1 / b$. Figure 8 displays the form factors for all $(K, f)$ parameter combinations at a low density $\left(\rho / \rho^{*}=0.1\right.$, panel (a)) and a high density beyond the percolation threshold $\left(\rho / \rho^{*}=4.0\right.$, panel (b)). Results for the intramolecularly cross-linked SCNPs are included for comparison. Far below the overlap concentration, the scaling exponents adopted by the chains with a higher bonding probability $p_{\mathrm{B} 0}$ at $\rho \rightarrow 0$ (higher $K$ and $f$ ) are systematically lower than for those with lower $p_{\mathrm{B} 0}$ (lower $K$ and $f$ ). At such concentrations, the intramolecular bonds are clearly dominant and a higher number of bonds (higher $p_{\mathrm{B}}$ ) lead to a stronger compaction (lower $\nu$ ) of the polymers, although the effect is moderate (changing from $\nu=0.59$ for the weakest bonds to $\nu=0.46$ for the permanent ones). This trend is consistent with experimental scaling exponents for reversible and irreversible SCNPs at high dilution. ${ }^{76}$

Far beyond the percolation threshold, the form factors of the reversible polymers of different bond strength and reactive fraction are nearly indistinguishable. Their scaling exponent $\nu$ $\approx 0.58$ is very close to the Flory exponent for self-avoiding conformations and clearly distinct from the exponent $\nu \sim 1 / 3$ characterizing the crumpling globular conformations of the intramolecularly cross-linked SCNPs in dense solutions. Such a big difference suggests that when concentrating the solution,
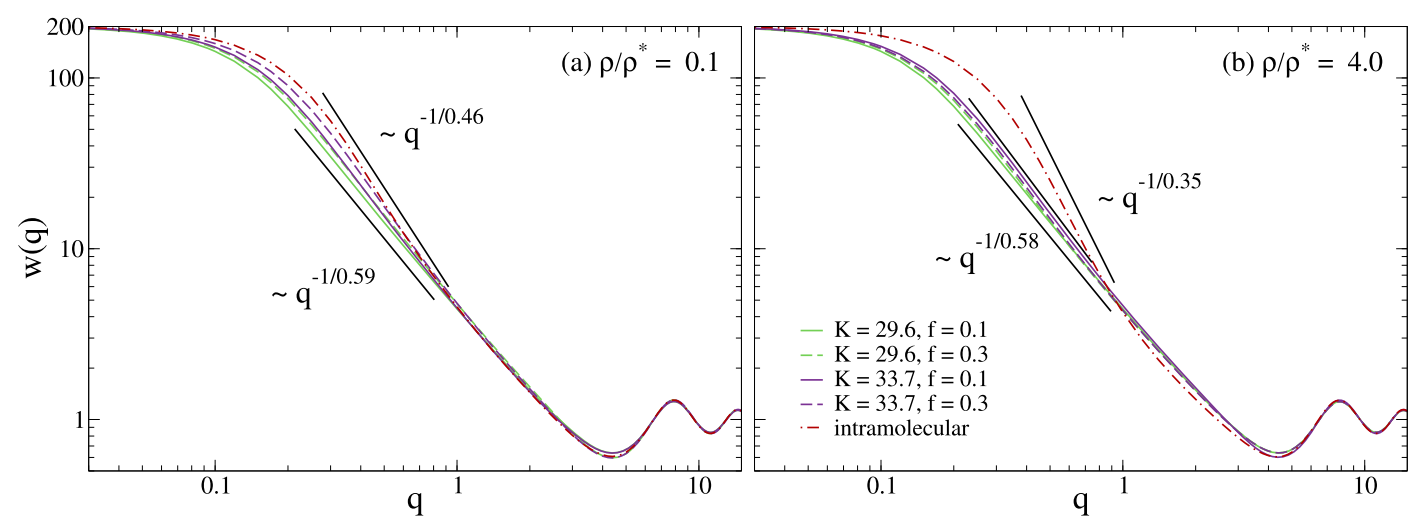

Figure 8. Intramolecular form factor $w(q)$ for all $(K, f)$ parameter combinations at densities (a) $\rho / \rho^{*}=0.1$ and (b) $\rho / \rho^{*}=4.0$, below and above the percolation threshold for all parameters, respectively. Dot-dashed lines are data for intramolecularly cross-linked SCNPs. Solid black lines are power laws representing the approximate scaling of $w(q)$ in the fractal regime. 
the long-range intramolecular loops of the intramolecularly bonded SCNPs are more likely to be exchanged for intermolecular bonds than the short-range ones (as discussed earlier, this exchange can be explained by a gain in conformational entropy via the opening of the long-range loops, see Figure 3). Moreover, the formation of new long intramolecular loops will involve concatenations with neighboring similar loops. Both mechanisms reduce the presence of the nonconcatenated long loops that are at the origin of the crumpled globular conformations of the intramolecularly crosslinked SCNPs in dense solutions. ${ }^{54,65}$

The large-scale structure of the system can be probed by calculating correlations between the macromolecular centersof-mass through the structure factor

$$
S_{\mathrm{CM}}(\mathbf{q})=\left\langle\frac{1}{N_{\mathrm{p}}} \sum_{j, k} \exp \left[\mathbf{i q} \cdot\left(\mathbf{r}_{\mathrm{CM}, j}-\mathbf{r}_{\mathrm{CM}, k}\right)\right]\right\rangle
$$

where $\mathbf{r}_{\mathrm{CM}, j}$ denotes the position of the center-of-mass of the macromolecule $j$. Figure 9 shows $S_{\mathrm{CM}}(q)$ for reversible chains

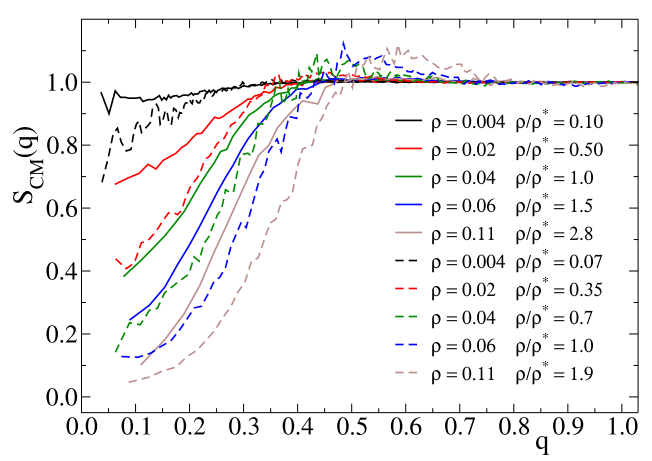

Figure 9. Structure factors of the macromolecular centers-of-mass for reversible chains (solid lines) with $K=33.7$ and $f=0.3$. Dashed lines represent data for the intramolecularly cross-linked SCNP counterparts. The same colors correspond to the same absolute values $(\rho)$ of the monomer density. The legend also indicates the normalized density $\left(\rho / \rho^{*}\right)$.

with $K=33.7$ and $f=0.3$ at various monomer densities. The polymers with other values of $(K, f)$ exhibit qualitatively the same behavior (not shown). For comparison, we include the corresponding data for intramolecularly cross-linked SCNPs at the same monomer densities. In the limit of $q \rightarrow 0$, the structure factor of the macromolecular centers-of-mass is proportional to the compressibility $\chi$ of the material

$$
\lim _{q \rightarrow 0} S_{\mathrm{CM}}(q)=\chi \rho k_{\mathrm{B}} T
$$

At the same monomer density, the low- $q$ limit of $S_{\mathrm{CM}}(q)$ is significantly and consistently lower for the solution formed by intramolecularly cross-linked SCNPs than for the network made up of reversibly bonded chains. The permanent crosslinks in the former prevent concatenation of loops and lead to less interpenetration of different SCNPs, rendering the system less compressible (lower $S(q=0)$ and $\chi$ ) than the reversible network at the same density. This difference in the degree of interpenetration is illustrated in Figure 10, which shows typical snapshots of a molecule and its 10 closest ones (in distance between centers-of-mass), at the same reduced density $\rho / \rho^{*}=$ 4 , in the reversible case $K=33, f=0.3$ and in the purely intramolecularly cross-linked system. (a)
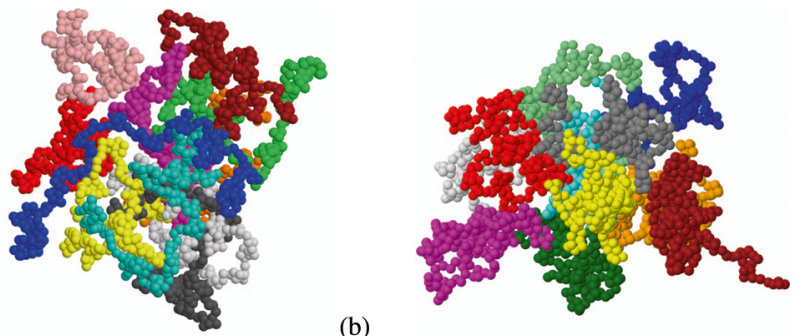

(b)

Figure 10. Snapshots of a molecule and its 10 closest ones (in distance between centers-of-mass), at the same reduced density $\rho / \rho^{*}$ $=4$, in the reversible case $K=33, f=0.3$ (a) and in the purely intramolecularly cross-linked system (b). Different molecules are represented with different colors.

Furthermore, in the investigated range of concentrations 0.1 $<\rho / \rho^{*}<4.4$, the structure factor shows no sign of phase separation or growing inhomogeneities (which would be manifested by a diverging or strongly growing $S(q)$ as $q \rightarrow 0)$. To assure that no phase separation is indeed intervening with gelation, we investigate density fluctuations in the system. To this end, the box is divided into sub-boxes of side $L_{\mathrm{s}}$ and the "local" monomer density $\phi$ is calculated within each of them. Density fluctuations are then defined by the parameter $\delta \phi^{2}=$ $\left\langle(\phi-\langle\phi\rangle)^{2}\right\rangle$, where the average is both taken over all subboxes and over various realizations (therefore the mean value of $\phi$ is identical to the macroscopic monomer density, $\langle\phi\rangle=$ $\rho)$. In the absence of correlations, fluctuations just behave as ${ }^{77}$ $\delta \phi^{2} \sim \rho$. Figure 11 shows the normalized quantity $\delta \phi^{2} / \rho$ as a

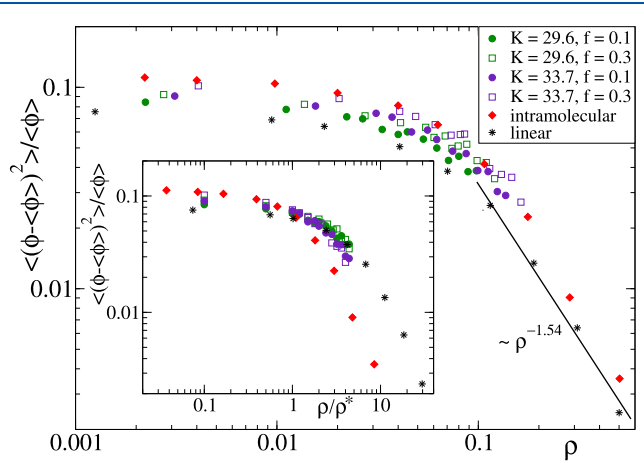

Figure 11. Average monomer density fluctuation, normalized by $\langle\phi\rangle$ $=\rho$ (see text) for a bin size of $L_{\mathrm{s}}=5$, for different values of the energy constant $K$ and reactive monomer fraction $f$. Results for the intramolecularly cross-linked SCNPs and for linear chains are included. Data in the main panel and in the inset are represented vs the absolute and the normalized $\left(\rho / \rho^{*}\right)$ monomer densities, respectively.

function of the concentration so that the effect of the correlations is reflected by deviations from a plateau. The data in the main panel are shown as a function of the absolute monomer density $\rho$. In order to higlight the different trends found below and above the overlap concentration, the inset shows the data as a function of $\rho / \rho^{*}$. The choice of the subbox size $L_{\mathrm{s}}$ influences the values of $\delta \phi^{2} / \rho$. If $L_{\mathrm{s}}$ is too small, fluctuations are inherently limited, while if it is too big, the phase occupying less volume might not be properly sampled. We varied $L_{\mathrm{s}}$ in a reasonable range $3 \leq L_{\mathrm{s}} \leq 20$ and the fluctation size showed the same dependence on the concentration irrespective of the specific $L_{s}$, the numerical 

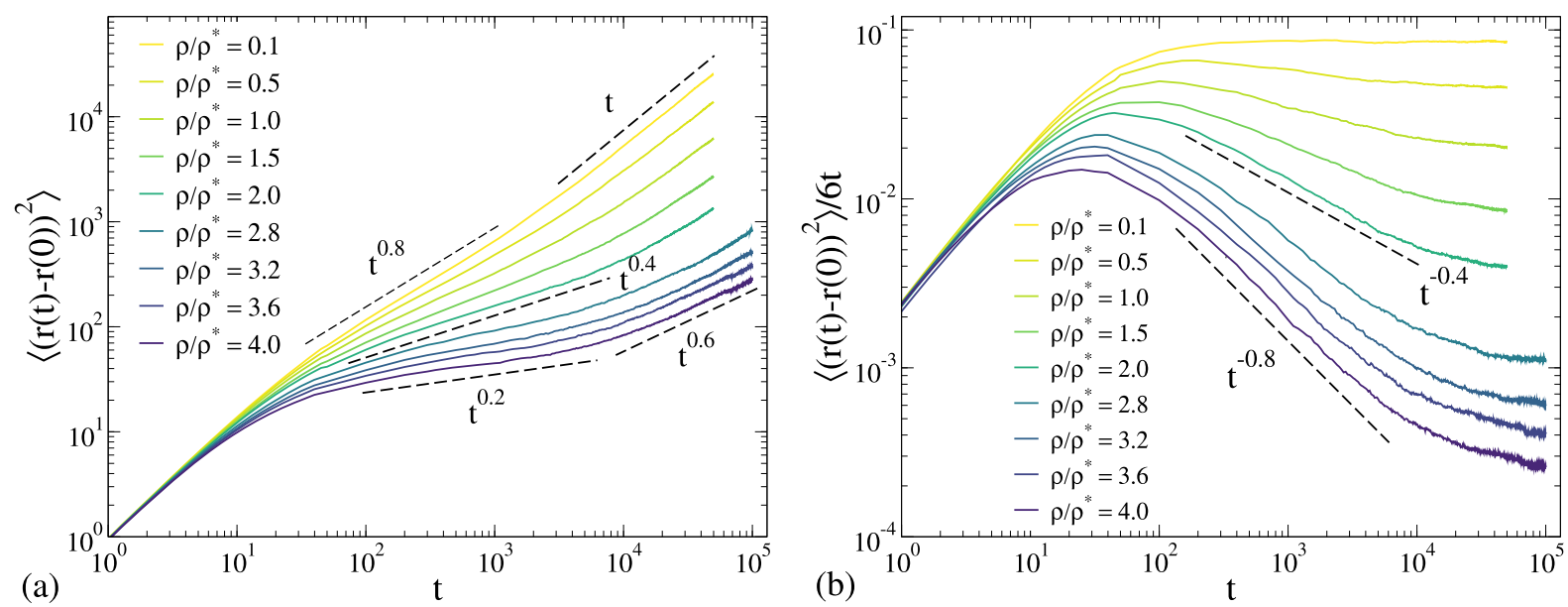

Figure 12. (a) Monomer mean-squared displacement for $K=33.7$ and $f=0.3$ at various densities. (b) As panel (a) for the molecular centers-ofmass. Data are normalized by $6 t$ to show that the diffusive regime (horizontal plateau in this representation) has been reached. Dashed lines in both panels indicate approximate power laws.

values just changing by a scaling factor. Data in Figure 11 were obtained using $L_{\mathrm{s}}=5$.

At high dilution, $\rho / \rho^{*} \ll 1, \delta \phi^{2} / \rho$ for the linear chains and the intramolecularly cross-linked SCNPs shows a plateau. As aforementioned, this result reflects vanishing correlations and is fully expected in such systems, due to both high dilution and the absence of bonding and aggregation. Above the overlap concentration $\left(\rho / \rho^{*}>1\right)$, under semidilute conditions, the low- $q$ limit of the structure factor of linear chains is expected to follow the relation ${ }^{56} S(0) / \rho \sim \delta \phi^{2} / \rho \sim \xi^{2}$, where $\xi$ is the correlation length. The portion of the linear chain within this length scale keeps its high-dilution scaling, that is, $\xi \sim g^{\nu_{\mathrm{F}}}$, with $g$ being the number of monomers of the chain in the correlation volume. Combining the former relations, we obtain

$$
\rho \sim \mathrm{g} / \xi^{3} \sim \xi^{1-3 \nu_{\mathrm{F}} / \nu_{\mathrm{F}}}
$$

and the concentration dependence of the fluctuations follows the power law

$$
\delta \phi^{2} / \rho \sim \xi^{2} \sim \rho^{-2 \nu_{\mathrm{F}} / 3 \nu_{\mathrm{F}}-1}
$$

Since $\nu_{\mathrm{F}}=0.588$, the fluctations in the solutions of linear chains are expected to decrease with the concentration as $\delta \phi^{2} /$ $\rho \sim \rho^{-1.54}$. This is confirmed in Figure 11. Similar results are found for the intramolecularly cross-linked SCNPs.

Remarkably, the solutions of reversibly cross-linking polymers show the same qualitative trends as the solutions of nonaggregating linear chains or intramolecularly crosslinked SCNPs, in particular, the plateau at low concentrations. Quantitative differences are found in the relative amplitudes of the fluctuations, which can be easily rationalized. For the same values of $L_{\mathrm{s}}$ and $\rho$, the amplitude of $\delta \phi^{2} / \rho$ is systematically higher as the molecular size decreases (see values of $R_{\mathrm{g} 0}$ in Table 1). Since all systems have the same molecular weight, smaller macromolecular sizes correspond to more compact objects, which at the same macroscopic density $\rho$ lead to higher values of $\delta \phi^{2}$ when they are distributed over the simulation box. In summary, data in Figure 11 are consistent with the results for the structure factors of Figure 9 and no signs of phase separation are found (otherwise consistently growing values of $\delta \phi^{2} / \rho$ with decreasing $\rho$ would be found instead of the plateau). Still, phase separation cannot be fully discarded-one should have in mind that fluctuations are limited by the simulation box. Unfortunately, the evaluation of the phase diagram for small densities is quite challenging and we cannot give a fully conclusive answer to this issue.

Dynamic Properties. After having discussed in detail, the structural properties of the networks formed through the reversible intermolecular bonds, we now shift our focus to the dynamics of the system. First, we calculate the mean-squared displacement of the individual monomers, $\operatorname{MSD}(t)=\left\langle\left(\mathbf{r}_{i}(t)-\right.\right.$ $\left.\left.\mathbf{r}_{i}(0)\right)^{2}\right\rangle$. Panel (a) of Figure 12 displays the MSD for the polymers with $K=33.7$ and $f=0.3$ at various densities. The panel (b) shows the corresponding results for the molecular centers-of-mass, divided by $6 t$ to show that the diffusive regime (horizontal asymptote in this representation) is reached. The other investigated reversible polymers display qualitatively the same trends (not shown). At short time scales, monomers diffuse freely without a density dependence. Upon increasing the density, a "soft" plateau appears in the MSD, marking a slowing down of the dynamics. ${ }^{78}$ The plateau can be described by a subdiffusive power law, MSD $\sim t^{x}$, with an exponent that decays with increasing the density $(x \approx 0.2$ at the highest simulated concentration). At high concentrations, when localization of the polymers is stronger, the square root of the value of the MSD in the plateau regime, which gives a measure of the localization length, is $\Delta=\sqrt{\mathrm{MSD}} \approx 5-8$, that is, of the order of the radius of gyration of the polymer. This is quite large, given that on average every third monomer along the chain (since $f=0.3$ ) is reactive and can potentially be engaged in a bond that strongly limits fluctuations. There are no significant dynamic heterogeneities in the system, as revealed by the close-to-Gaussian shape of the van Hove self-correlation functions $\left(G_{s}(r, t)\right)$ of the monomers (see representative data in Figure 13 for $K=33.7, f=0.3$ at the density $\left.\rho / \rho^{*}=4.0\right)$.

The large localization length and the progressive slowing down reflected in Figure 12 are clear signatures of approaching a gel transition. The values of $D$, obtained as MSD/ $6 t$ of the molecular centers-of-mass at the end of the simulation time window, are presented in Figure 14 for different parameters $(K$, $f)$. Not surprisingly, the diffusivity shows a sharp decay when the concentration is increased beyond the overlap density and at every time, most of the polymers are bonded to the percolating cluster. For the highest $K$ and $f$, the decay can be 


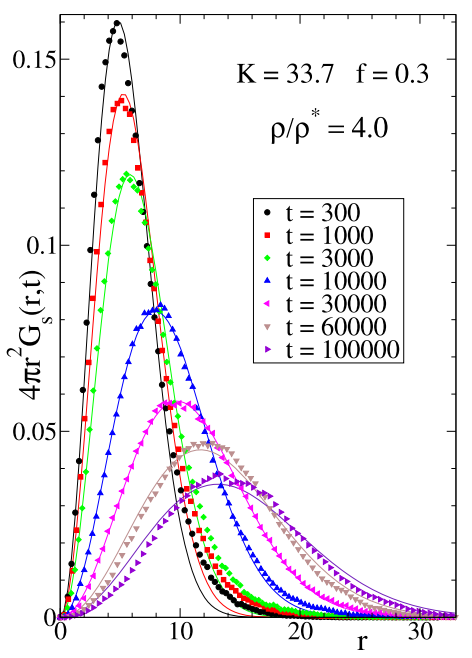

Figure 13. Van Hove self-correlation function of the monomers at different times, for the case $K=33.7, f=0.3$ at $\rho / \rho^{*}=4.0$. Symbols: simulation data; lines: fits to Gaussian functions (note the phase factor $\left.4 \pi r^{2}\right)$.

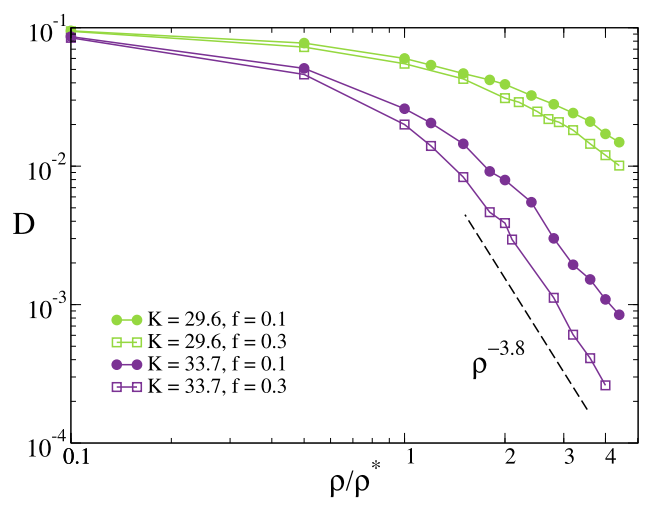

Figure 14. Density dependence of the diffusivity, calculated from the long-time limit of the mean-squared displacement of the molecular centers-of-mass, for different combinations of $K$ and $f$.

approximately described by a power law $\mathrm{D} \sim \rho^{-3.8}$. This is much higher than the exponents for, for example, concentrated solutions of entangled linear chains ${ }^{56}(x=2.33)$, highlighting the effect of the gelation process in the polymer dynamics.
Finally, we investigate the bond dynamics and the reorganization of the percolating cluster once it is formed. We first analyze whether the lifetime of intermolecular bonds is affected by gelation and whether cooperativity plays a role in the formation of intermolecular bonds. To this end, we calculate the intermolecular bond self-correlation

$$
S_{\text {inter }}(t)=\frac{\left\langle B_{i j}(t) B_{i j}(0)\right\rangle}{\left\langle B_{i j}(0)\right\rangle^{2}}
$$

where $B_{i j}(t)=1$ if the reactive monomers $i$ and $j$ (belonging to different chains) form a bond at time $t$ and this has never been broken since $t=0$. If the bond has been broken at least once, then $B_{i j}(t)=0$. Furthermore, we calculate the self-correlation of the cluster

$$
S_{\text {cluster }}(t)=\frac{\left\langle M_{i}(t) M_{i}(0)\right\rangle}{\left\langle M_{i}(0)\right\rangle^{2}}
$$

where $M_{i}(t)=1$ if polymer $i$ has been a member of the percolating cluster at all times $0 \leq t^{\prime} \leq t$ and $M_{i}(t)=0$ otherwise. As such, the relaxation of $S_{\text {cluster }}(t)$ provides a measure for the average dissociation time from the cluster. Figure 15 displays the intermolecular bond self-correlation as well as the cluster self-correlation for $f=0.3$ and for the two investigated bond strengths. In both cases, the intermolecular bond self-correlation is not affected by changes in the density and can be well-described by an exponential decay, $S_{\text {inter }}(t) \sim$ $\mathrm{e}^{-t / \tau}$. This exponential relaxation is also found for the intramolecular bonds (not shown), demonstrating that bond breaking is purely governed by temperature (as the bond strength is akin to an inverse temperature) and bonding is not cooperative (stretched exponentials would be otherwise observed).

On the other hand, the cluster self-correlation (weakly nonexponential) is strongly affected by density. For $K=33.7$, at high densities, the percolating cluster becomes so stable that almost no polymers leave it within the simulation time window. This, however, does not mean that the cluster does not rearrange. Indeed, within the simulation time scale, the polymers are able to diffuse distances of more than $3 R_{\mathrm{g} 0} \approx 26$ even at $\rho / \rho^{*}=4.0$ (see Figure 12). An average of 6 intermolecular connections per chain at this concentration (see Figure 5) allows the polymers to move through the cluster, breaking and reforming bonds, without ever detaching from it.
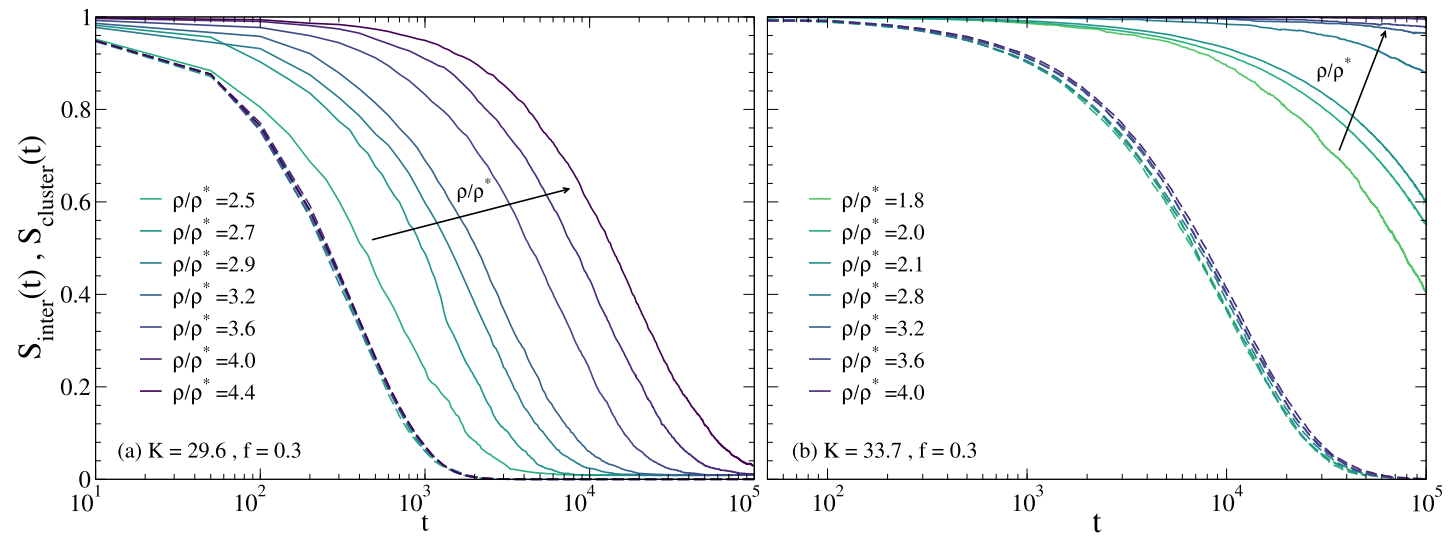

Figure 15. Intermolecular bond $\left(S_{\text {inter }}(t)\right.$, dashed lines $)$ and cluster $\left(S_{\text {cluster }}(t)\right.$, solid lines $)$ self-correlation function for $f=0.3$ and $(\mathrm{a}) K=29.6,(\mathrm{~b})$ $K=33.7$. Only densities in which a percolating cluster is at least transiently present are considered. 
This stability of the cluster along with its potential to rearrange could lead to an interesting behavior under external stresses, which should be investigated in future work. Furthermore, it is not clear whether intermolecular or intramolecular bonds would break first under shear, potentially leading to an interesting viscoelastic response.

At intermediate densities, a significant number of chains (see Figure 15b) detach from the infinite cluster from time to time and moves through the system before getting reabsorbed in the cluster again. We monitor each polymer that leaves the percolating cluster at some time during the simulation and compute the time it spends since it leaves it until it is reattached. The results of this analysis are shown in Figure 16.

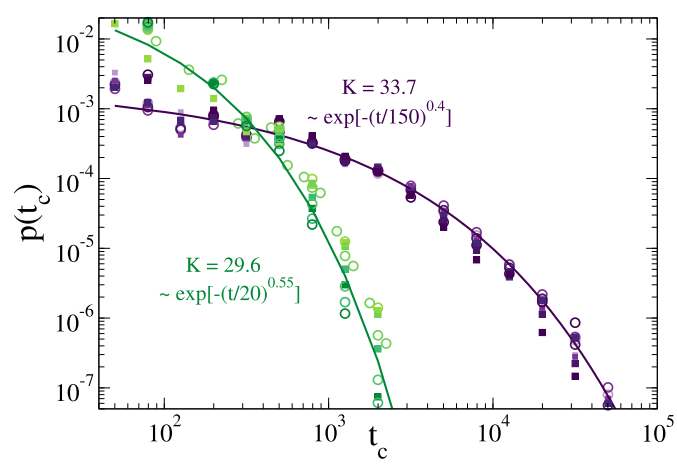

Figure 16. Distribution of times a polymer spends outside the systemspanning cluster for $K=29.6$ (green symbols) and $K=33.7$ (purple symbols). Data correspond to both $f=0.1$ (squares) and $f=0.3$ (circles) at various densities above the percolation threshold. Darker colors correspond to higher densities. Solid lines are fits to stretched exponential functions. Data are shown for the following ranges of densities: $3.2 \leq \rho / \rho^{*} \leq 4.4(K=29.6, f=0.1) ; 2.8 \leq \rho / \rho^{*} \leq 4.4(K$ $=29.6, f=0.3) ; 1.5 \leq \rho / \rho^{*} \leq 3.2(K=33.7, f=0.1)$; and $1.2 \leq \rho / \rho^{*}$ $\leq 2.0(K=33.7, f=0.3)$. As shown in other figures, for $K=33.7$, we have performed simulations at higher concentrations. However, at such concentrations, the number of chains that performed excursions out of the percolating cluster was not large enough to have reasonable statistics, and the corresponding distributions $p\left(t_{c}\right)$ are not displayed.

Surprisingly, the distributions of times spent outside the percolating cluster are essentially independent of the number of reactive monomers and of the density of the solution. We find a universal distribution solely dependent on the bond strength $K$ that follows a stretched exponential $p\left(t_{c}\right) \sim \mathrm{e}^{-\left(t / \tau_{c}\right) \beta}$, with $\beta=0.55$ for $K=29.6$ and $\beta=0.40$ for $K=33.7$. As shown in Figure 14, the diffusivity strongly depends on the density, for example, for the case $K=33.7$ and $f=0.1$, the diffusivity decays by an order of magnitude in the range $1.5 \leq \rho / \rho^{*} \leq$ 3.2 , while in that same range, $p\left(t_{c}\right)$ is at most marginally dependent on the concentration with no systematic trend.

As can be seen in Figure 16, for $K=33.7$, most of the free motions between detachment and reattachment occur within time scales $t_{c}<10^{4}\left(p\left(t_{c}\right)\right.$ has decayed 2 orders of magnitude at that time), which as seen in the analysis of the MSD correspond to displacements of at most the polymer size. At the relaxation time $\tau_{\mathrm{c}}=150$, the MSD is still in the soft plateau regime, that is, in that time scale, the typical free chain is still localized in the region of its last detachment. In other words, free chains do not perform long flights until they find an unbonded reactive group in the percolating cluster and are reattached to it. This result suggests that the reattachment time is not controlled by the characteristic diffusivity over large scales (which indeed is strongly affected by crowding) but rather by the time it takes to break a bond (essentially controlled by the energy scale $K \varepsilon$ ) so that the new unbonded monomers in the free chain can explore their counterparts in the percolating cluster through conformational fluctuations.

\section{CONCLUSIONS}

We have investigated the gel formation of linear polymer chains decorated with functional groups with the ability to form reversible bonds. For this purpose, we have employed Langevin dynamics simulations and a bond potential that mimics reversible covalent bonds for the synthesis of reversible SCNPs. We have studied in detail the competition between intra- and intermolecular bonding as a function of the concentration, finding that the replacement of intramolecular links by intermolecular ones prohibits a straightforward treatment of the system via Wertheim's theory and its use for elucidating the complete phase diagram. The formation of a system-spanning cluster takes place above the overlap concentration and seems to converge to it by increasing the bond strength and fraction of reactive groups.

Surprisingly, the introduction of intermolecular bonds induces a nonmonotonic dependence of the radius of gyration on the density for high bond strengths, while the polymers shrink monotonically for lower bond strengths. In all the cases, the variation of the polymer size with respect to high dilution conditions is at most $5 \%$, even at 4 times the overlap concentration. Concomitantly, the scaling properties of the polymer conformations are weakly perturbed, and beyond the percolation transition, they can be approximately described by self-avoiding random walk statistics irrespective of the bond strength. These results for the intramolecular conformations in the reversible systems are rather different from those in crowded solutions and melts of intramolecularly cross-linked SCNPs with no intermolecular bonds. In such systems, the purely intramolecular permanent cross-links prevent concatenation of loops, leading to a collapse of purely entropic origin to crumpled globular conformations, in order to optimize packing in the solution. In the reversible case, the intramolecular loops, creating topological interactions between two chains, can be broken and concatenated later with other loops when a new intramolecular bond is formed. Under such conditions, the SCNPs become more open and penetrable, and the microsegregration seen in intramolecularly cross-linked SCNPs is circumvented, making the system significantly more compressible.

Finally, we have demonstrated that the dynamics of the system display the soft caging regime expected for gel-forming materials. The reorganization dynamics of the percolating cluster is mostly mediated by the diffusion, through breaking and formation of bonds, of chains that, without detaching from the percolating cluster, are able to explore distances of several times their size. A remarkable universal behavior is found: the time a polymer spends out of the percolating cluster is independent of the density and solely depends on the bond lifetime, which suggests that reattachment to the percolating cluster is limited by the availability of free reactive groups and not by the diffusion of the free chain.

In conclusion, our results present valuable preliminary insights into the gelling process of reversibly cross-linking polymers with randomly distributed functional groups. The competition between intra- and intermolecular bonds leads to complex structural rearrangements purely governed by 
entropical contributions. Systems in which such a competition is present have not been studied extensively in the literature to this date. We hope that our results will motivate further research efforts in this direction. On the other hand, the possibility of combining advanced functions (catalysis, luminescence, etc.), polymer flexibility, dynamic bonds, and assembly into equilibrium gels make reversible SCNPs potentially attractive systems as building blocks for the design of smart materials with self-healing properties.

\section{ASSOCIATED CONTENT}

\section{SI Supporting Information}

The Supporting Information is available free of charge at https://pubs.acs.org/doi/10.1021/acs.macromol.0c02670.

Static and dynamic observables for a different set of parameters of the reversible bonding potential (PDF)

\section{AUTHOR INFORMATION}

\section{Corresponding Author}

Angel J. Moreno - Centro de Física de Materiales (CSIC, UPV/EHU) and Materials Physics Center MPC, E-20018 San Sebastián, Spain; Donostia International Physics Center (DIPC), E-20018 San Sebastián, Spain; 이이.org/00000001-9971-0763; Email: angeljose.moreno@ehu.es

\section{Authors}

Maud Formanek - Centro de Física de Materiales (CSIC, UPV/EHU) and Materials Physics Center MPC, E-20018 San Sebastián, Spain; Sainsbury Laboratory, University of Cambridge, CB2 1LR Cambridge, U.K.

Lorenzo Rovigatti - Dipartimento di Fisica, Università di Roma La Sapienza, IT-00185 Roma, Italy; CNR-ISC Uos Sapienza, IT-00185 Roma, Italy; 이이이.org/0000-00015017-2829

Emanuela Zaccarelli - CNR-ISC Uos Sapienza, IT-00185 Roma, Italy; Dipartimento di Fisica, Università di Roma La Sapienza, IT-00185 Roma, Italy; (1) orcid.org/0000-00030032-8906

Francesco Sciortino - Dipartimento di Fisica, Università di Roma La Sapienza, IT-00185 Roma, Italy; (1) orcid.org/ 0000-0002-2418-2713

Complete contact information is available at:

https://pubs.acs.org/10.1021/acs.macromol.0c02670

\section{Notes}

The authors declare no competing financial interest.

\section{ACKNOWLEDGMENTS}

We acknowledge support from the projects PGC2018-094548B-I00 (MCIU/AEI/FEDER, UE) and IT-1175-19 (Basque Government, Spain). M.F. acknowledges travel grants from Materials Physics Center (San Sebastián, Spain). We acknowledge support of the publication fee by the CSIC Open Access Publication Support Initiative through its Unit of Information Resources for Research (URICI).

\section{REFERENCES}

(1) Lyon, C. K.; Prasher, A.; Hanlon, A. M.; Tuten, B. T.; Tooley, C. A.; Frank, P. G.; Berda, E. B. A brief user's guide to single-chain nanoparticles. Polym. Chem. 2015, 6, 181-197.
(2) Gonzalez-Burgos, M.; Latorre-Sanchez, A.; Pomposo, J. A. Advances in single chain technology. Chem. Soc. Rev. 2015, 44, 61226142.

(3) Liu, Y.; Pauloehrl, T.; Presolski, S. I.; Albertazzi, L.; Palmans, A. R. A.; Meijer, E. W. Modular synthetic platform for the construction of functional single-chain polymeric nanoparticles: from aqueous catalysis to photosensitization. J. Am. Chem. Soc. 2015, 137, 1309613105 .

(4) Altintas, O.; Barner-Kowollik, C. Single-chain folding of synthetic polymers: a critical update. Macromol. Rapid Commun. 2016, 37, 29-46.

(5) Hanlon, A. M.; Lyon, C. K.; Berda, E. B. What is next in singlechain nanoparticles? Macromolecules 2016, 49, 2-14.

(6) Single-Chain Polymer Nanoparticles: Synthesis, Characterization, Simulations, and Applications; Pomposo, J. A., Ed.; John Wiley \& Sons: Weinheim, Germany, 2017.

(7) Rubio-Cervilla, J.; González, E.; Pomposo, J. A. Advances in Single-Chain Nanoparticles for Catalysis Applications. Nanomaterials 2017, 7, 341.

(8) De-La-Cuesta, J.; González, E.; Pomposo, J. A. Advances in Fluorescent Single-Chain Nanoparticles. Molecules 2017, 22, 1819.

(9) Pia, A.; Kröger, P.; Paulusse, J. M. Single-chain polymer nanoparticles in controlled drug delivery and targeted imaging. $J$. Controlled Release 2018, 286, 326-347.

(10) Rothfuss, H.; Knöfel, N. D.; Roesky, P. W.; Barner-Kowollik, C. Single-Chain Nanoparticles as Catalytic Nanoreactors. J. Am. Chem. Soc. 2018, 140, 5875-5881.

(11) Kröger, A. P. P.; Komil, M. I.; Hamelmann, N. M.; Juan, A.; Stenzel, M. H.; Paulusse, J. M. J. Glucose Single-Chain Polymer Nanoparticles for Cellular Targeting. ACS Macro Lett. 2019, 8, 95101.

(12) Frisch, H.; Tuten, B. T.; Barner-Kowollik, C. Macromolecular Superstructures: A Future Beyond Single Chain Nanoparticles. Isr. J. Chem. 2020, 60, 86-99.

(13) Verde-Sesto, E.; Arbe, A.; Moreno, A. J.; Cangialosi, D.; Alegría, A.; Colmenero, J.; Pomposo, J. A. Single-chain nanoparticles: opportunities provided by internal and external confinement. Mater. Horiz. 2020, 7, 2292-2313.

(14) Chen, J.; Li, K.; Shon, J. S. L.; Zimmerman, S. C. Single-Chain Nanoparticle Delivers a Partner Enzyme for Concurrent and Tandem Catalysis in Cells. J. Am. Chem. Soc. 2020, 142, 4565-4569.

(15) Mavila, S.; Eivgi, O.; Berkovich, I.; Lemcoff, N. G. Intramolecular cross-linking methodologies for the synthesis of polymer nanoparticles. Chem. Rev. 2016, 116, 878-961.

(16) Sanchez-Sanchez, A.; Pomposo, J. A. Single-Chain Polymer Nanoparticles via Non-Covalent and Dynamic Covalent Bonds. Part. Part. Syst. Charact. 2014, 31, 11-23.

(17) Artar, M.; Huerta, E.; Meijer, E. W.; Palmans, A. R. A. SequenceControlled Polymers: Synthesis, Self-Assembly, and Properties; ACS Publications, 2014; pp 313-325.

(18) Altintas, O.; Barner-Kowollik, C. Single Chain Folding of Synthetic Polymers by Covalent and Non-Covalent Interactions: Current Status and Future Perspectives. Macromol. Rapid Commun. 2012, 33, 958-971.

(19) Seo, M.; Beck, B. J.; Paulusse, J. M. J.; Hawker, C. J.; Kim, S. Y. Polymeric nanoparticles via noncovalent cross-linking of linear chains. Macromolecules 2008, 41, 6413-6418.

(20) Foster, E. J.; Berda, E. B.; Meijer, E. W. Metastable supramolecular polymer nanoparticles via intramolecular collapse of single polymer chains. J. Am. Chem. Soc. 2009, 131, 6964-6966.

(21) Terashima, T.; Mes, T.; De Greef, T. F. A.; Gillissen, M. A. J.; Besenius, P.; Palmans, A. R. A.; Meijer, E. W. Single-Chain Folding of Polymers for Catalytic Systems in Water. J. Am. Chem. Soc. 2011, 133, $4742-4745$

(22) Hosono, N.; Gillissen, M. A. J.; Li, Y.; Sheiko, S. S.; Palmans, A. R. A.; Meijer, E. W. Orthogonal self-assembly in folding block copolymers. J. Am. Chem. Soc. 2012, 135, 501-510.

(23) Burattini, S.; Colquhoun, H. M.; Fox, J. D.; Friedmann, D.; Greenland, B. W.; Harris, P. J. F.; Hayes, W.; Mackay, M. E.; Rowan, 
S. J. A self-repairing, supramolecular polymer system: healability as a consequence of donor-acceptor $\pi-\pi$ stacking interactions. Chem. Commun. 2009, 6717-6719.

(24) Appel, E. A.; Dyson, J.; del Barrio, J.; Walsh, Z.; Scherman, O. A. Formation of single-chain polymer nanoparticles in water through host-guest interactions. Angew. Chem., Int. Ed. 2012, 51, 4185-4189.

(25) Wang, F.; Pu, H.; Che, X. Voltage-responsive single-chain polymer nanoparticles via host-guest interaction. Chem. Commun. 2016, 52, 3516-3519.

(26) Huh, J.; Park, H. J.; Kim, K. H.; Kim, K. H.; Park, C.; Jo, W. H. Giant Thermal Tunability of the Lamellar Spacing in BlockCopolymer-Like Supramolecules Formed from Binary-End-Functionalized Polymer Blends. Adv. Mater. 2006, 18, 624-629.

(27) Hofmeier, H.; Schubert, U. S. Combination of orthogonal supramolecular interactions in polymeric architectures. Chem. Commun. 2005, 2423-2432.

(28) Gohy, J.-F.; Hofmeier, H.; Alexeev, A.; Schubert, U. S. Aqueous micelles from supramolecular graft copolymers. Macromol. Chem. Phys. 2003, 204, 1524-1530.

(29) Tuten, B. T.; Chao, D.; Lyon, C. K.; Berda, E. B. Single-chain polymer nanoparticles via reversible disulfide bridges. Polym. Chem. 2012, 3, 3068-3071.

(30) Murray, B. S.; Fulton, D. A. Dynamic covalent single-chain polymer nanoparticles. Macromolecules 2011, 44, 7242-7252.

(31) Sanchez-Sanchez, A.; Fulton, D. A.; Pomposo, J. A. pHresponsive single-chain polymer nanoparticles utilising dynamic covalent enamine bonds. Chem. Commun. 2014, 50, 1871-1874.

(32) He, J.; Tremblay, L.; Lacelle, S.; Zhao, Y. Preparation of polymer single chain nanoparticles using intramolecular photodimerization of coumarin. Soft Matter 2011, 7, 2380-2386.

(33) Frank, P. G.; Tuten, B. T.; Prasher, A.; Chao, D.; Berda, E. B. Intra-chain photodimerization of pendant anthracene units as an efficient route to single-chain nanoparticle fabrication. Macromol. Rapid Commun. 2014, 35, 249-253.

(34) Whitaker, D. E.; Mahon, C. S.; Fulton, D. A. Thermoresponsive dynamic covalent single-chain polymer nanoparticles reversibly transform into a hydrogel. Angew. Chem., Int. Ed. 2013, 52, 956-959.

(35) Zaccarelli, E. Colloidal gels: equilibrium and non-equilibrium routes. J. Phys.: Condens. Matter 2007, 19, 323101.

(36) Corezzi, S.; Fioretto, D.; Puglia, D.; Kenny, J. M. Light scattering study of vitrification during the polymerization of model epoxy resins. Macromolecules 2003, 36, 5271-5278.

(37) Del Gado, E.; Fierro, A.; de Arcangelis, L.; Coniglio, A. A unifying model for chemical and colloidal gels. Europhys. Lett. 2003, 63, 1.

(38) Saika-Voivod, I.; Zaccarelli, E.; Sciortino, F.; Buldyrev, S. V.; Tartaglia, P. Effect of bond lifetime on the dynamics of a short-range attractive colloidal system. Phys. Rev. E: Stat., Nonlinear, Soft Matter Phys. 2004, 70, 041401.

(39) Rovigatti, L.; Sciortino, W. K. Self and collective correlation functions in a gel of tetrahedral patchy particles. Mol. Phys. 2011, 109, 2889-2896.

(40) Groenewold, J.; Kegel, W. Colloidal cluster phases, gelation and nuclear matter. J. Phys.: Condens. Matter 2004, 16, S4877.

(41) Sciortino, F.; Tartaglia, P.; Zaccarelli, E. One-dimensional cluster growth and branching gels in colloidal systems with shortrange depletion attraction and screened electrostatic repulsion. J. Phys. Chem. B 2005, 109, 21942-21953.

(42) Ruiz-Franco, J.; Zaccarelli, E. On the Role of Competing Interactions in Charged Colloids with Short-Range Attraction. Annu. Rev. Condens. Matter Phys. 2021, 12, 51.

(43) Zaccarelli, E.; Buldyrev, S.; La Nave, E.; Moreno, A.; SaikaVoivod, I.; Sciortino, F.; Tartaglia, P. Model for reversible colloidal gelation. Phys. Rev. Lett. 2005, 94, 218301.

(44) Segre, P.; Prasad, V.; Schofield, A.; Weitz, D. Glasslike kinetic arrest at the colloidal-gelation transition. Phys. Rev. Lett. 2001, 86, 6042 .
(45) Manoharan, V. N.; Elsesser, M. T.; Pine, D. J. Dense packing and symmetry in small clusters of microspheres. Science 2003, 301, 483-487.

(46) Cho, Y.-S.; Yi, G.-R.; Lim, J.-M.; Kim, S.-H.; Manoharan, V. N.; Pine, D. J.; Yang, S.-M. Self-organization of bidisperse colloids in water droplets. J. Am. Chem. Soc. 2005, 127, 15968-15975.

(47) Biffi, S.; Cerbino, R.; Bomboi, F.; Paraboschi, E. M.; Asselta, R.; Sciortino, F.; Bellini, T. Phase behavior and critical activated dynamics of limited-valence DNA nanostars. Proc. Natl. Acad. Sci. U.S.A. 2013, 110, 15633-15637.

(48) Rovigatti, M. S.; Smallenburg, F.; Romano, F.; Sciortino, F. Gels of DNA nanostars never crystallize. ACS Nano 2014, 8, 35673574.

(49) Wertheim, M. S. Fluids with highly directional attractive forces. I. Statistical thermodynamics. J. Stat. Phys. 1984, 35, 19-34.

(50) Wertheim, M. Fluids with highly directional attractive forces. II. Thermodynamic perturbation theory and integral equations. J. Stat. Phys. 1984, 35, 35-47.

(51) Bianchi, E.; Largo, J.; Tartaglia, P.; Zaccarelli, E.; Sciortino, F. Phase diagram of patchy colloids: Towards empty liquids. Phys. Rev. Lett. 2006, 97, 168301.

(52) Whitesides, G. M.; Boncheva, M. Beyond molecules: Selfassembly of mesoscopic and macroscopic components. Proc. Natl. Acad. Sci. U.S.A. 2002, 99, 4769-4774.

(53) Glotzer, S. C. Some Assembly Required. Science 2004, 306, 419-420.

(54) Moreno, A. J.; Lo Verso, F.; Arbe, A.; Pomposo, J. A.; Colmenero, J. Concentrated Solutions of Single-Chain Nanoparticles: A Simple Model for Intrinsically Disordered Proteins under Crowding Conditions. J. Phys. Chem. Lett. 2016, 7, 838-844.

(55) Kremer, K.; Grest, G. S. Dynamics of entangled linear polymer melts: A molecular-dynamics simulation. J. Chem. Phys. 1990, 92, 5057-5086.

(56) Rubinstein, M.; Colby, R. H. Polymer Physics; Oxford University Press: Oxford, U.K., 2003; Vol. 23.

(57) Moreno, A. J.; Lo Verso, F.; Sanchez-Sanchez, A.; Arbe, A.; Colmenero, J.; Pomposo, J. A. Advantages of Orthogonal Folding of Single Polymer Chains to Soft Nanoparticles. Macromolecules 2013, 46, 9748-9759.

(58) Formanek, M.; Moreno, A. J. Effects of precursor topology and synthesis under crowding conditions on the structure of single-chain polymer nanoparticles. Soft Matter 2017, 13, 6430-6438.

(59) Formanek, M.; Moreno, A. J. Single-Chain Nanoparticles under Homogeneous Shear Flow. Macromolecules 2019, 52, 1821-1831.

(60) Formanek, M.; Moreno, A. J. Crowded Solutions of SingleChain Nanoparticles under Shear Flow, 2019. arXiv:1910.01425.

(61) Smallenburg, F.; Sciortino, F. Liquids more stable than crystals in particles with limited valence and flexible bonds. Nat. Phys. 2013, 9, 554.

(62) Locatelli, E.; Handle, P. H.; Likos, C. N.; Sciortino, F.; Rovigatti, L. Condensation and Demixing in Solutions of DNA Nanostars and Their Mixtures. ACS Nano 2017, 11, 2094-2102.

(63) Izaguirre, J. A.; Catarello, D. P.; Wozniak, J. M.; Skeel, R. D. Langevin stabilization of molecular dynamics. J. Chem. Phys. 2001, 114, 2090-2098.

(64) Rudnick, J.; Gaspari, G. The Shapes of Random Walks. Science $1987,237,384-389$.

(65) Gonzalez-Burgos, M.; Arbe, A.; Moreno, A. J.; Pomposo, J. A.; Radulescu, A.; Colmenero, J. Crowding the Environment of SingleChain Nanoparticles: A Combined Study by SANS and Simulations. Macromolecules 2018, 51, 1573-1585.

(66) Sukumaran, S. K.; Grest, G. S.; Kremer, K.; Everaers, R. Identifying the primitive path mesh in entangled polymer liquids. $J$. Polym. Sci., Part B: Polym. Phys. 2005, 43, 917-933.

(67) Roldán-Vargas, S.; Smallenburg, F.; Kob, W.; Sciortino, F. Phase diagram of a reentrant gel of patchy particles. J. Chem. Phys. 2013, 139, 244910. 
(68) Marshall, B. D.; Ballal, D.; Chapman, W. G. Wertheim's association theory applied to one site patchy colloids: Beyond the single bonding condition. J. Chem. Phys. 2012, 137, 104909.

(69) Sciortino, F. Entropy in Self-assembly. Riv. Nuovo Cimento 2019, 42, 511-548.

(70) Sciortino, F.; Zhang, Y.; Gang, O.; Kumar, S. K. CombinatorialEntropy-Driven Aggregation in DNA-Grafted Nanoparticles. ACS Nano 2020, 14, 5628-5635.

(71) Flory, P. J. Molecular size in three dimensional polymers. I. Gelation. II. Tri-functional branching units. III. Tetrafunctional branching units. J. Am. Chem. Soc. 1941, 63, 3083.

(72) Stockmayer, W. H. Theory of molecular size distribution and gel formation in branched-chain polymers. J. Chem. Phys. 1943, 11, $45-55$.

(73) Stockmayer, W. H. Theory of molecular size distribution and gel formation in branched polymers II. General cross linking. J. Chem. Phys. 1944, 12, 125-131.

(74) Stauffer, D.; Aharony, A. Introduction to Percolation Theory; Taylor \& Francis: London, 1992.

(75) Halverson, J. D.; Lee, W. B.; Grest, G. S.; Grosberg, A. Y.; Kremer, K. Molecular dynamics simulation study of nonconcatenated ring polymers in a melt. I. Statics. J. Chem. Phys. 2011, 134, 204904.

(76) Pomposo, J. A.; Rubio-Cervilla, J.; Moreno, A. J.; Lo Verso, F.; Bacova, P.; Arbe, A.; Colmenero, J. Folding Single Chains to SingleChain Nanoparticles via Reversible Interactions: What Size Reduction Can One Expect? Macromolecules 2017, 50, 1732-1739.

(77) Barrat, J. L.; Hansen, J. P. Basic Concepts for Simple and Complex Liquids; Cambridge University Press: Cambridge, U.K., 2003.

(78) One should note that the absence, prior to the plateau, of an intermediate Rouse-like regime (MSD $\left.\sim t^{0.5}\right)$ is due to the use of a low friction constant in order to speed up equilibration and sampling. 